\title{
In situ nozzle reservoir thermometry by laser-induced grating spectroscopy in the HELM free-piston reflected shock tunnel
}

\author{
C. Selcan ${ }^{1} \cdot$ T. Sander $^{1} \cdot$ Ch. Mundt ${ }^{1}$
}

Received: 30 October 2019 / Accepted: 30 November 2020 / Published online: 9 January 2021

(c) The Author(s) 2021

\begin{abstract}
Experimental determination of test gas caloric quantities in high-enthalpy ground testing is impeded by excessive pressure and temperature levels as well as minimum test timescales of short-duration facilities. Yet, accurate knowledge of test gas conditions and stagnation enthalpy prior to nozzle expansion is crucial for a valid comparison of experimental data with numerical results. To contribute to a more accurate quantification of nozzle inlet conditions, an experimental study on nonintrusive in situ measurements of the post-reflected shock wave stagnation temperature in a large-scale free-piston reflected shock tunnel is carried out. A series of 20 single-shot temperature measurements by resonant homodyne laser-induced grating spectroscopy (LIGS) is presented for three low-/medium-enthalpy conditions (1.2-2.1 MJ/kg) at stagnation temperatures 1100-1900 K behind the reflected shock wave. Prior limiting factors resulting from impulse facility recoil and restricted optical access to the high-pressure nozzle reservoir are solved, and advancement of the optical set-up is detailed. Measurements in air agree with theoretical calculations to within $1-15 \%$, by trend reflecting greater temperatures than full thermo-chemical equilibrium and lesser temperatures than predicted by ideal gas shock jump relations. For stagnation pressures in the range 9-22 MPa, limited influence due to finite-rate vibrational excitation is conceivable. LIGS is demonstrated to facilitate in situ measurements of stagnation temperature within full-range ground test facilities by superior robustness under high-pressure conditions and to be a useful complement of established optical diagnostics for hypersonic flows.
\end{abstract}

Keywords Free-piston shock tunnel $\cdot$ Hypersonic nozzle reservoir $\cdot$ Stagnation enthalpy $\cdot$ High-temperature $\cdot$ High pressure $\cdot$ Laser diagnostics $\cdot$ LIGS $\cdot$ LITA

\section{Introduction}

Evaluation of experimental test data in high-enthalpy and hypersonic ground testing requires complementary flowfield computations in order to obtain flow quantities of interest that are not available to direct measurement [1]. For this purpose, the accurate definition of boundary conditions is vital. This is similarly true for freestream quantities at the

Communicated by S. O’Byrne.

This paper is based on work that was presented at the 32 nd International Symposium on Shock Waves, Singapore, July 14-19, 2019.

C. Selcan

claudio.selcan@unibw.de

1 Institute for Thermodynamics, Bundeswehr University Munich, Werner-Heisenberg-Weg 39, 85577 Neubiberg, Germany nozzle exit, which serve as inlet boundary conditions to test geometry computations and are themselves typically determined by computational fluid dynamics (CFD) analysis of the nozzle expansion or numerical rebuilding methods, e.g., via ex situ stagnation heat flux measurements [2], see Fig. 1.

Typically, total flow enthalpy is determined by quasi one-dimensional (1-D) numerical codes such as L1d, ESTCj [3], or Kasimir [4] based on incident shock Mach number and assuming normal shock relations [5] as well as quasi-steady conditions thereafter, as long as test conditions are properly tailored. In practice, physical three-dimensional (3-D) phenomena are more complex due to the presence of the shock tube wall boundary layer and endwall-reflected shock bifurcation such that post-reflected conditions in a reflected shock tunnel can vary over time [6]. In effect, the time interval of constant post-shock temperature (and species concentration) can be smaller than quasi-steady pressure duration, i.e., test time, even in tailored interface mode due to premature driver gas 


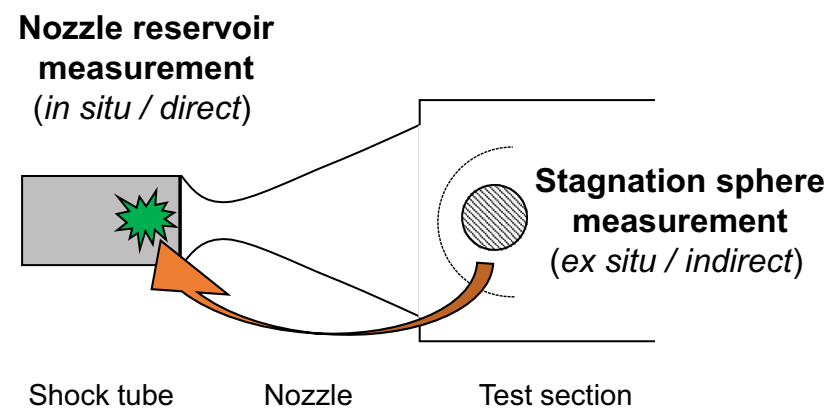

Fig. 1 Principle of in situ measurements in the nozzle reservoir and ex situ measurements in the freestream of a short-duration facility

contamination [7, 8]. Hornung [9] suggested operation in an overtailored mode to be sometimes necessary.

Therefore, particularly in the case of impulse facilities of short duration such as reflected shock tunnels and expansion tubes, knowledge of caloric quantities of the stagnated test gas prior to nozzle expansion is highly desirable in order to accurately determine nozzle inlet conditions and eventually increase the accuracy of calculated freestream quantities [10].

A non-trivial way to achieve this are in situ measurements within the nozzle reservoir, which can be a useful complement to established ex situ rebuilding approaches [2]. Yet, as much as direct experimental acquisition of test gas temperature and enthalpy is highly desirable, investigation of high-enthalpy flows by conventional probes is strongly restricted by thermal, mechanical, and temporal limitations. Adverse effects such as aerodynamic blockage and heat loss due to thermal radiation are well known. Optical techniques, in contrast, are well suited for measurements under severe conditions due to their non-intrusiveness and absence of thermal and mechanical restrictions. Yet, in the light of the severely limited test time, excessive pressure, temperature, and density levels in ground test facilities as well as closely restricted optical access to the highly loaded nozzle reservoir, requirements for suitable optical diagnostics are closely defined:

1. Steady test times below $10 \mathrm{~ms}$ and limited number of test runs per day demand high repetition rate $(\mathrm{kHz}-\mathrm{MHz})$ lasers or single-shot capability.

2. Geometrical alignment of laser beam path is to be robust to axial facility displacement due to mechanical recoil and reaction force, particularly in free-piston tunnels.

3. Limited geometrical access to the reservoir section and spectral properties of the window material are to match needs of the optical technique.

4. Strong gas luminosity and spurious background radiation at high-enthalpy conditions significantly complicate the detection of weak light signals, favouring the use of coherent techniques wherever possible [1].

Evidently, the strength of some established laser techniques for hypersonic flows is increasingly limited when to be applied to the highly loaded nozzle reservoir section of a free-piston shock tunnel.

\section{Measurement technique}

\subsection{Application of optical diagnostics}

In the following, the viability and potential of application of several well-established optical diagnostics to the nozzle reservoir of a short-duration impulse facility is discussed.

Spontaneous Raman spectroscopy is attractive due to a relatively simple optical set-up of a single laser beam, fundamental single-pulse capability, absence of collisional quenching effects, and restriction to resonant excitation; however, it suffers from a severely weak signal intensity and incoherent signal scattering [11]. Despite relatively inexpensive spectral analysis, it is thus not suited to acquire singleshot measurements under harsh high-temperature conditions, all the more in the presence of strong background luminosity further lowering the signal-to-noise ratio (SNR).

Coherent anti-Stokes Raman scattering (CARS) offers higher signal intensity due to a coherent signal beam and single-shot capability when using a broadband dye laser to generate a full CARS spectrum of Raman resonances. As with incoherent Raman scattering, CARS necessitates wavelength-resolved spectroscopic detection of a full signal spectrum and, due to its nonlinear nature, requires high pump laser energy. As gas temperature is determined indirectly from spectral shape, the major disadvantage of CARS is posed by expensive data reduction, mandating for extensive spectral calculations by convolution of Stokes/pump laser profile and spectrometer-detector instrument function [10]. This spectroscopic modelling is further sensitive to background noise, Raman transition linewidth (line profile), and precise spectral location and thus affected by pressure line broadening to be accounted for [11]. Still, CARS was applied to shock tunnel experiments to great advantage in the past, particularly in the low-pressure, low-density freestream at the nozzle exit. Boyce et al. [10] measured vibrational/ rotational temperatures in the freestream and spherical shock layer at pressures below 3 bar and attributed accuracy and precision of single-pulse CARS up to $10 \%$ to be limited by pulse-to-pulse amplitude and phase fluctuations of the dye laser profile's mode structure. Pulford et al. [12] measured shock tunnel freestream temperature of up to $3000 \mathrm{~K}$ at a scramjet model inlet at pressures around 1 bar with a singleshot precision up to $11.4 \%$. According to the authors' best 
knowledge, broadband CARS has been successfully applied at the overall highest pressure of $6.5 \mathrm{MPa}$ so far: Grisch et al. [13] measured temperatures up to $2000 \mathrm{~K}$, reporting pressure line broadening to become increasingly difficult to capture/ model at simultaneously high pressure and temperature. Thus, CARS application to quantitative single-shot temperature determination in the high-pressure nozzle reservoir is deemed difficult by the authors.

Another potential technique is laser-induced fluorescence (LIF) which-due to its inherent linear nature of a given species' resonant excitation and despite isotropic (incoherent) emission-features signal intensity of magnitudes stronger than Raman scattering [11]. As for spontaneous Raman scattering, a principle strength of LIF is its potential to acquire two-dimensional (2-D) spatial distributions of temperature and species concentration which is yet not deemed to provide much additional information in shock tube flows of uniform centerline development. Quantitative analysis of spectrally resolved LIF signals is significantly affected by radiative decay rates of excited states due to predissociation, photoionization, and collisional quenching which are difficult to account for-the latter being particularly relevant to shock tube flow due to high pressure and density $[12,14]$. Furthermore, rotational and vibrational energy transfer processes are to be modelled by adequate rate models [11]. A variant avoiding this problem of collisional quenching, known as laser-induced predissociation fluorescence (LIPF), was used by Sutton et al. [15] to measure vibrational temperatures within a shock tunnel testbody shock layer; however, the severely low fluorescence yield, i.e., signal intensity of LIPF-in comparison with regular single-pulse LIF-resulted in experimental uncertainty as high as $10-18.6 \%$. As the fluorescence yield of LIF noticeably decreases with increasing pressure and as the signal suffers from diffuse background radiation and flow luminosity, the technique is similarly not deemed applicable to a highpressure shock tunnel reservoir [1]. Moreover, the general strength of 2-D imaging techniques such as PLIF is best used to advantage within flowfields of inherent spatial variation, as occurs within the freestream downstream of the nozzle exit [16], whereas the flowfield within the shock tube and nozzle reservoir of test facilities is generally considered spatially uniform and thus does not demand for spatial imaging.

Line-of-sight integration diagnostics such as emission spectroscopy and tunable diode laser spectroscopy (TDLAS), relying on sampling of species' self-emitted radiation spectral intensity as well as intensity decrease in irradiated light due to stimulated absorption as it transects the measurement volume, respectively, offer a relatively simple set-up. For emission spectroscopy, quantitative analysis of the sampled spectrum necessitates proper calibration and detailed knowledge of the related species (mainly in ground state or weakly excited electronic levels) to study the gas chemistry involved. In general, description of upper state population in relation to ground state via Boltzmann distribution holds for thermodynamic equilibrium [1], such that application to the shock tunnel reservoir at high pressure and density is in principle viable. Yet, quantitative analysis towards temperature measurements, either by spectral line shape or by line strength ratio of two transitions, is foreseeably impaired by collisional line broadening at very high pressures. As absorption along the line of sight cannot be separated from the emitted spectrum, indirect quantitative analysis requires detailed computation of predicted radiation spectra, thus making analysis very complicated. To the best knowledge of the authors, emission spectroscopy [17] and TDLAS [18] have so far only been used for temperature measurements at very low pressures, i.e., at atmospheric pressure or below.

Against this background, laser-induced grating spectroscopy (LIGS) has recently emerged as a promising method to complement the range of established measurement techniques for shock tube flows. LIGS is also known as laserinduced thermo-acoustics (LITA) in the literature. By providing for greater (sub-microsecond) temporal resolution, high robustness under adverse conditions, single-shot capability for temperature determination as well as minimum need for optical access, LIGS has been recently identified as a viable and robust laser diagnostic technique. It is deemed particularly suited for an application to in situ measurements within the nozzle reservoir of hypersonic impulse facilities [19-21]. LIGS is fundamentally related to degenerate fourwave mixing (DFWM), a technique suggested by Eckbreth [11] for application in high-interference, high-pressure environments of restricted optical access. Yet, DFWM typically uses non-resonant excitation for temperature measurements only and is gradually impaired by an excitation of resonant gratings [22]. Single-shot measurements by LIGS, in contrast, do not demand for a multiplex approach of broadband excitation, e.g., wavelength scanning, and spectrally resolved detection by frequency-resolved detectors but utilize a single-mode laser and time-resolved detector instead and can likewise use resonant and non-resonant excitation to advantage [23, 24].

\subsection{LIGS application}

Regarding the capability of deterministic, time-accurate, pointwise measurement of post-shock sound speed, i.e., temperature, and fluid velocity, i.e., Mach number, by a single laser pulse, in conjunction with straightforward signal analysis and data reduction, LIGS proves to be a versatile and robust laser diagnostic technique. As such, it is considered well suited to complement more established methodologies of shock tube research. In the light of excessive pressure levels prevalent in high-enthalpy ground testing, the positive 
correlation of resonant and non-resonant LIGS signal intensity with fluid pressure is considered particularly advantageous. Whereas quantitative measurements by a range of competitive techniques are increasingly impeded at elevated pressures-resultant from a decaying signal strength due to higher collisional quenching rates $[19,25]$ — the signal intensity $I$ of LIGS benefits from greater pressures $p$ and rises according to (1) [26]:

$I \propto p^{2} \cdot T^{-3.4}$

where $T$ denotes the temperature. Coherent signal beam nature is most beneficial to yield high detection efficiency even in the harsh high-enthalpy environment of shock tunnels, further aggravated by spurious extrinsic light interference from background radiation and metallic impurities encountered [12].

Sources of uncertainty (particularly single-shot fluctuation) for coherent laser beam techniques in general, such as beam steering and defocusing (induced by gradual beam deflection due to density gradients within the traversed highly compressible flowfield), are known from CARS measurements [13] and likewise apply to LIGS [27]. A strong feature of LIGS, which distinguishes it from a range of established intensity-based diagnostics, is the pure frequency-based signal character of LIGS thermometry that reflects its superior robustness to various error sources of quantitative signal intensity deterioration, such as collisional quenching or background luminosity. LIGS is also insensitive to fluctuations in laser intensity. Similarly, once the coherent signal beam is detected and successfully isolated from spurious background radiation, specular reflections, and undesired probe and pump beams, via optical bandpass filters and pinhole apertures, respectively, quantitative data reduction is straightforward and unambiguous. This is particularly true in comparison with intensity-based, spatially resolved techniques such as PLIF. Using a photomultiplier tube (PMT), i.e., single-point time-resolved detector, is advantageous in comparison with multichannel detectorsas employed in broadband CARS - by avoiding nonlinear instrument response and without the general need for spectrally resolved detection by high-resolution spectrometers as employed in multiplex approaches. PMTs offer variable gain to amplify very low-intensity signals (as applies to LIGS) and provide for fast (sub-nanoseconds) time response. In contrast to techniques utilizing broadband excitation, spectral intensity distributions of the dye laser, i.e., fluctuating power density and mode structure - as applies to CARScan be entirely neglected [13]. Whereas the LIGS signal intensity increases favourably at higher pressures, quantitative thermometry by spectral methods becomes increasingly difficult, e.g., CARS temperature accuracy depends on collisional linewidth due to pressure broadening that is to be accounted for and-especially at simultaneously high temperatures where Doppler broadening becomes further strong - can only be obtained from linewidth model extrapolation [13].

First successful application of LIGS to the transient test time regime of a small-size double-diaphragm shock tube was demonstrated by Sander et al. [19], measuring temperatures behind the incident and reflected shock by resonant and non-resonant homodyne LIGS, up to $840 \mathrm{~K}$ and at $2 \mathrm{MPa}$. Comparison of measurements to 1-D shock jump relations (ideal gas) based on incident shock Mach number showed maximum single-shot relative deviation of $7-8 \%$ from theory. High precision of gas temperature measurement by LIGS at Mach numbers $1.37-2.20$ was indicated by a standard deviation of maximum 3\%, for a shock Mach number of 1.91 . The shock tube was spatially fixed in a foundation such that no recoil occurred to induce deflection and laser beam misalignment throughout the experiment. In a similar small-sized conventional, spatially fixed shock tube, Förster et al. [28] presented simultaneous single-shot temperature and velocity measurements by non-resonant heterodyne LIGS. Experiments in nitrogen and argon at shock Mach numbers $1.67-1.96$ were conducted behind the incident and reflected shock at post-shock temperature and pressure up to $1000 \mathrm{~K}$ and $4.3 \mathrm{MPa}$, respectively. With measurement precision of gas temperature on the order of $3 \%$ and the potential for simultaneous accurate determination of flow Mach number, with a relative deviation as low as $1.4 \%$, the authors reasoned LIGS to be well suited for measurements of shock-heated flows.

Recently, Selcan et al. [20] and Altenhöfer et al. [21] successfully demonstrated measurement technique feasibility of LIGS towards in situ determination of total temperature conditions of a full-scale, hypersonic ground test facility for the first time. Using resonant excitation $\left(\mathrm{NO}_{2}\right.$-seeded) homodyne LIGS at $593 \mathrm{~nm}$ for a representative low-enthalpy test condition at $1.2 \mathrm{MJ} / \mathrm{kg}$, post-reflected shock stagnation temperatures of $900-1100 \mathrm{~K}$ at pressures from 5 to $6 \mathrm{MPa}$ were quantified by single-shot measurements in the nozzle reservoir of the piston-driven reflected shock tunnel HELM. Measurements were conducted in air, at incident shock Mach numbers up to 2.6. Comparing two alternative signal processing procedures, the authors found a time-domain least-squares fit of the temporal LIGS signal to provide a higher precision of 3.04\%, in comparison with $3.88 \%$ for a frequency-domain analysis by fast Fourier transform (DFT). As for accuracy, mean temperature relative deviation for a single test condition is found to be as low as $-1.82 \%$ and $-0.61 \%$ for the DFT and time fit, respectively, indicating single-shot temperature measurements by LIGS to be well correlated and to yield good agreement with 1-D inviscid theory in the low-enthalpy test regime. Thereby, measurement technique robustness for single-shot stagnation temperature determination and its general 
applicability to large-scale short-duration ground test facilities has been proven beyond prior use in conventional shock tube configurations and at the overall highest test gas conditions.

Based on prior investigations in the HELM shock tunnel, the current article describes extension of the measurement range of operation conditions to nozzle reservoir pressures, temperatures, and enthalpies of up to $21 \mathrm{MPa}, 1900 \mathrm{~K}$, and $2.2 \mathrm{MJ} / \mathrm{kg}$, respectively. Thereby, according to the best knowledge of the authors, single-shot measurements presented in the following have been conducted at the overall highest test conditions in shock tube experiments so far, where LIGS was applied for means of non-intrusive, i.e., remote, thermometry.

\subsection{Measurement principle}

Laser-induced grating spectroscopy (LIGS) is a coherent diagnostic technique which exploits the nonlinear generation of a local density pattern (acoustic waves) in a fluid due to induction of a local electric/intensity field as resulting from light interference of two pump laser beams. Probing of the grating by means of a third incident continuous wave (cw) interrogation beam results in the diffraction off the interference pattern under the Bragg angle: the scattered signal beam is guided to a single-point detector where information on the measurement volume is conveyed by frequency modulation of signal intensity oscillation.

As two short-pulsed pump laser beams intersect at a shallow angle, an interference pattern or electric/intensity field grating is seen to span the intersection region in the test fluid, i.e., sample volume. Figure 2 depicts grating formation resulting from intersection of two coherent pulsed pump beams (wave vectors $k_{1}$ and $k_{2}$, wavelength $\lambda_{\text {pump }}$ ) with crossing full angle $\Theta$. The grating vector $q$ is perpendicular to the fringe pattern, whereas the grating constant $\Lambda$ is determined by the pump laser wavelength $\lambda_{\text {pump }}$ and crossing angle $\Theta$ according to (2):

$\Lambda=\frac{\lambda_{\text {pump }}}{2 \cdot \sin (\Theta / 2)}$.

In the following, two opto-acoustic effects, termed electrostriction and thermalization, lead to the development of a congruent density interference pattern. Electrostriction describes the tendency of polarizable molecules to move towards regions of high electric field intensity and is proportional to the real part of the gas susceptibility [29]. Thermalization in contrast is a multiple-step collisional quenching process, proportional to the imaginary part of the gas susceptibility [30]. As the gas is excited by driver laser light at resonant wavelength, molecules absorb incident radiation and are excited to higher electronic states. Pressure-dependent inelastic collisions in turn convert part of the excited state energy into molecular kinetic, rotational, or vibrational energy, which is finally dissipated as heat by elastic molecular collisions equilibrating molecular velocities, thus inducing a rise in temperature and change in particle density. The generated density perturbation field is composed of two plane acoustic (sound) waves moving in opposite direction, in parallel to the grating vector. Sound wave propagation gives rise to alternating constructive and destructive interference, resulting in a temporal oscillation of local fluid density. Accordingly, the refraction index of test gas in the measurement volume varies in time with a characteristic oscillation (scattering) frequency $f_{\mathrm{M}}$. It is defined according to the spatial dimension of the density grating (i.e., fringe spacing) and sound wave propagation within the fluid, according to (3):

$f_{\mathrm{M}}=c \cdot \frac{a}{\Lambda}$

where $a$ denotes the speed of sound of the test gas. Constant $c$ yields: $c=2$ for electrostrictive (non-resonant) and $c=1$ for thermal (resonant) grating excitation such that signal frequency equals either the onefold or twofold of the socalled Brillouin frequency. Accordingly, signal oscillation frequency varies by a factor of 2 which serves for unambiguous distinction of contributions by scattering off a thermal (resonant) or electrostrictive (non-resonant) grating [23].

As a cw probe beam (wavelength $\lambda_{\text {probe }}$ ) intersects the measurement volume under the Bragg angle $\varphi$, the probe beam is diffracted off the temporally oscillating grating. This scattered signal beam is coherent in nature and conveys information on the density grating due to frequency modulation of the signal intensity, according to the oscillation
Fig. 2 Schematic of the interference grating and laser beam geometry

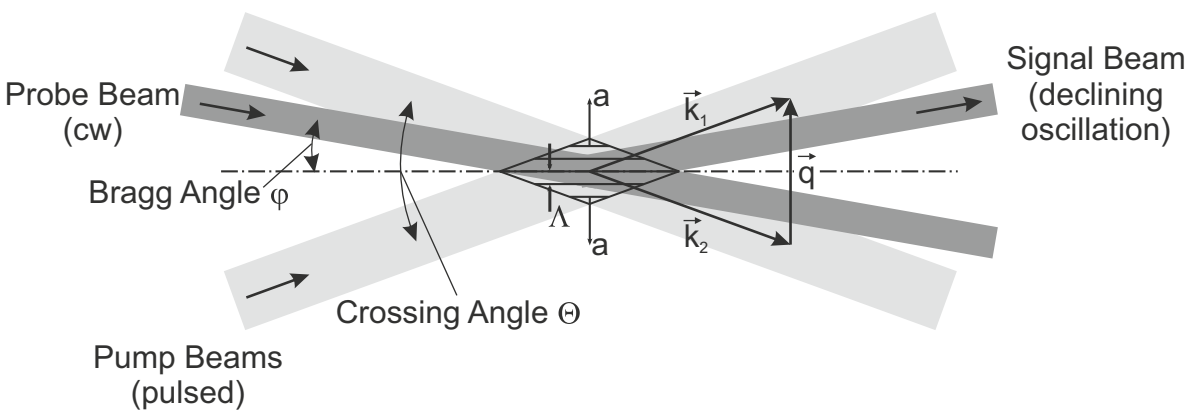


frequency as resulting from sound wave propagation. The Bragg angle condition for Bragg angle $\varphi$ is given by (4):

$2 \cdot \sin (\varphi)=\frac{\lambda_{\text {probe }}}{\Lambda}$.

When acquired by a detector (e.g., photomultiplier), frequency-domain analysis of the scattered signal beam via a discrete fast Fourier transform (DFT) yields the oscillation frequency $f_{\mathrm{M}}$ of the induced density grating. Thus, provided the grating constant $\Lambda$ and nature of the grating (electrostrictive or thermal) are known a priori, the speed of sound within the measurement volume can be calculated from (3). For a known gas composition, local temperature of the single-point sample volume spanned by the interference pattern can be calculated from the associated speed of sound.

Due to heat conduction (thermal diffusion) and acoustic damping (sound wave attenuation), density grating intensity and thus probe beam diffraction decrease with time. Accordingly, the scattered signal beam intensity is characterized by a temporally damped oscillation. A more detailed account on the physical background of laser-induced dynamic light scattering, density grating formation as well as analytical postulation of signal temporal evolution by means of the linearized equations of hydrodynamics and light scattering is given by Cummings et al. [29] and Schlamp et al. [31].

While single-shot temperature measurements by LIGS can be likewise conducted exploiting resonant and non-resonant wavelength excitation, thermal, i.e., resonant, gratings are considered particularly well suited for measurement in the adverse environment of a compression-heated, reflected shock tunnel. Precisely, wavelength-independent electrostrictive, i.e., non-resonant, gratings require very large pump beam pulse energies (>100 mJ) [23] which are, however, prohibitive in the present case of the laser focal point being situated near the optical access windows and shallow beam intersection angles. Resonant excitation of thermal gratings by contrast does not require high pump beam pulse energies [23] but benefits primarily from higher pressures and associated collisional quenching rates due to rapid thermalization [22]. Thereby, loss in signal intensity due to low pulse energies is compensated by the use of selective seeding. Accordingly, electrostrictive (non-resonant) excitation is not deemed applicable by the authors such that LIGS measurements via thermal (resonant) gratings are regarded much more robust and particularly attractive — even at low pump energies dictated by the optical window damage threshold-for application in shock tunnels of significantly limited optical access and high stagnation pressure and density. The intrinsic formation of nitric dioxide $\left(\mathrm{NO}_{2}\right)$ as active, i.e., resonantly excited, species at adequate concentrations in the post-shock regime-particularly at higher test gas conditions-will yield increased signal intensities, sufficient for measurement at elevated pressures and temperatures when air is used as test gas, see (1). However, in the case of test facilities where the imperative of low pump beam pulse energies (as imposed by the optical windows' damage threshold) was significantly alleviated, measurement by electrostrictive (non-resonant) LIGS will-in principle be likewise possible. For both thermal (resonant) and electrostrictive (non-resonant) gratings alike, a decrease in signal amplitude due to elevated temperatures of shortduration facilities is more than compensated by the pressure rise in the shock-processed test gas, eventually yielding a rise in signal intensity at increasingly ground-test-relevant conditions, see (1). In fact, based on signal intensity, as the pressure ratio across the normal shock will always be significantly greater than the corresponding temperature ratio, LIGS is projected to yield sufficient signal intensity even for probing of high-enthalpy conditions in reflected shock tunnels.

As for the detection scheme, homodyne LIGS detection-as being used in the present study — describes the use of a single signal beam (scattered off the optical grating) for measurements of local sound speed and temperature. Alternatively, a so-called heterodyne approach uses a second interrogation beam as local oscillator (i.e., reference beam) not passing the measurement volume but being mixed with the signal beam from the measurement volume prior to detection. Interference of both signal and reference beams enables additional quantification of the local bulk fluid velocity (single component) from the oscillation beat frequency and Doppler shift — thus enabling direct, simultaneous measurement of local flow Mach number via sound speed and fluid velocity; compare Förster et al. [28]. The present study solely applies the homodyne approach as quantification of fluid residual velocity components in the high-vorticity, post-reflected shock state 5 is not considered of interest.

\section{Experimental facility}

\subsection{HELM shock tunnel}

Experimental stagnation temperature measurements by LIGS are conducted within the nozzle reservoir section of the free-piston reflected shock tunnel facility HELM at the Bundeswehr University Munich. The (medium size) hypersonic wind tunnel was commissioned in 2010 and provides means for ground testing of high-speed flows by a free-piston configuration of the driver section [32]. Rapid compression and heating of the driver gas upstream of the primary (steel) diaphragm enable high ratios of pressure and speed of sound of the driver and test gas, respectively. Resultant strong shock waves generated upon main diaphragm rupture facilitate high stagnation enthalpies and pressures (greater 
than $20 \mathrm{MK} / \mathrm{kg}$ and $200 \mathrm{MPa}$, respectively) upon incident shock wave reflection from the endwall of the driven section $[33,34]$. When operating in reflected shock tunnel configuration, a thin Mylar diaphragm is employed as solid end to reach post-shock stagnation conditions before melting and giving way to the downstream Laval and expansion nozzle flow towards the test chamber. For operation in shock tube operation, a fixed endwall piece (steel) is being used.

The facility has a total length of $39 \mathrm{~m}$, with a length of the compression tube (CT) and shock tube (ST) of $21 \mathrm{~m}$ and $10 \mathrm{~m}$, respectively. An aluminium compression piston of weight $57.6 \mathrm{~kg}$ is used for the low-enthalpy experiments presented herein. A schematic of the facility is shown in Fig. 3.

Post-reflected shock wave (state 5) stagnation temperature measurements by LIGS are conducted in the nozzle reservoir section at the shock tube endwall and Laval nozzle inlet. The orthogonal measurement plane is situated $40 \mathrm{~mm}$ upstream of the endwall (secondary diaphragm) and is optically accessible by radial bores equipped with sapphire windows.

LIGS measurements are carried out using both opposing, horizontal bores $\left(180^{\circ}\right.$ from one another) to guide pump, probe, and signal beams into the measurement volume and again out of the shock tube section to the PMT detector. Both pump beams interfere at intersection angle $\Theta$, giving rise to an acoustic grating locally at the shock tube centerline, see Fig. 4.

A fast-response piezoelectric pressure transducer (type PCB 111A22) coincident, i.e., coplanar, with the measurement volume is used to acquire pressure traces and test gas stagnation pressure throughout the experiment. The pressure transducer is thermally insulated against heat flux influence by a thin layer of high-temperature silicone. Incident/reflected shock wave speed and thus Mach number $M_{\mathrm{s}}$ are determined by the difference in shock-front time of arrival, as obtained from two adjacent, flush-mounted wall pressure gauges (PCB 111A22), placed $700 \mathrm{~mm}$ from one another and situated closely upstream of the measurement plane, see Fig. 3. Facility operation and test gas conditions are monitored by compression and shock tube dynamic pressure traces. Data are acquired by a transient recorder with 14-bit ADC amplitude digitization at a sampling rate of $500 \mathrm{kHz}$.

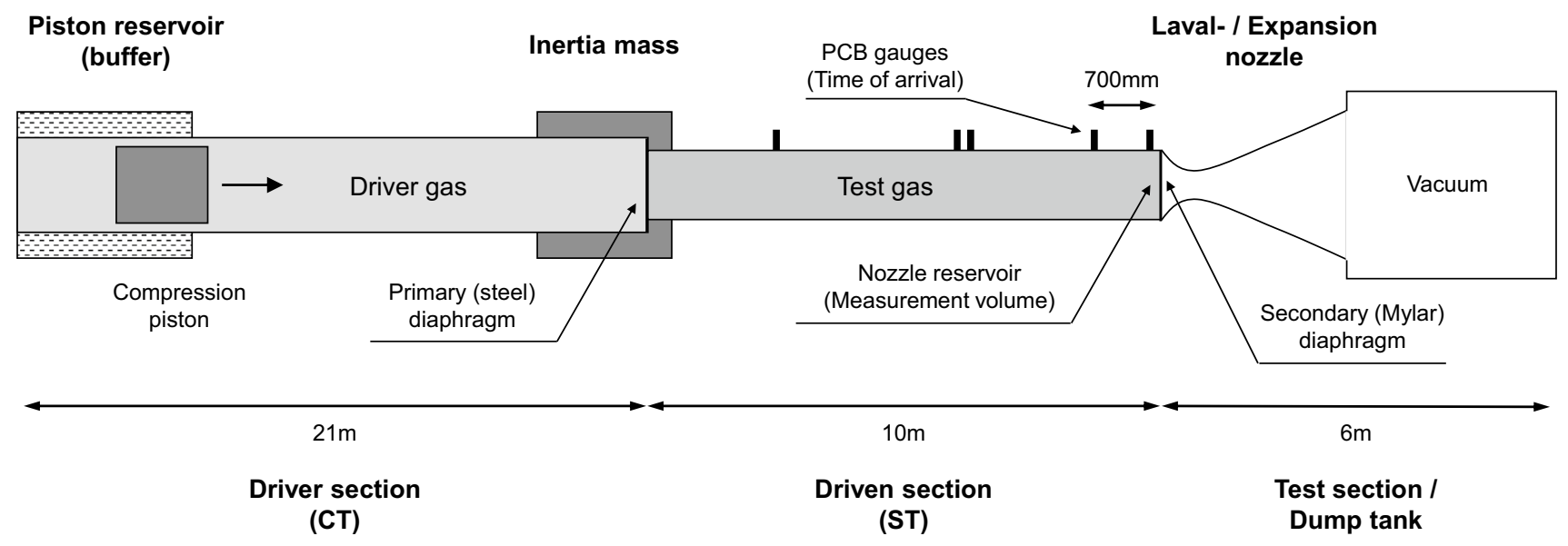

Fig. 3 Schematic of the HELM high-enthalpy shock tunnel facility

Fig. 4 Cross-sectional view of the shock tube nozzle reservoir and measurement volume

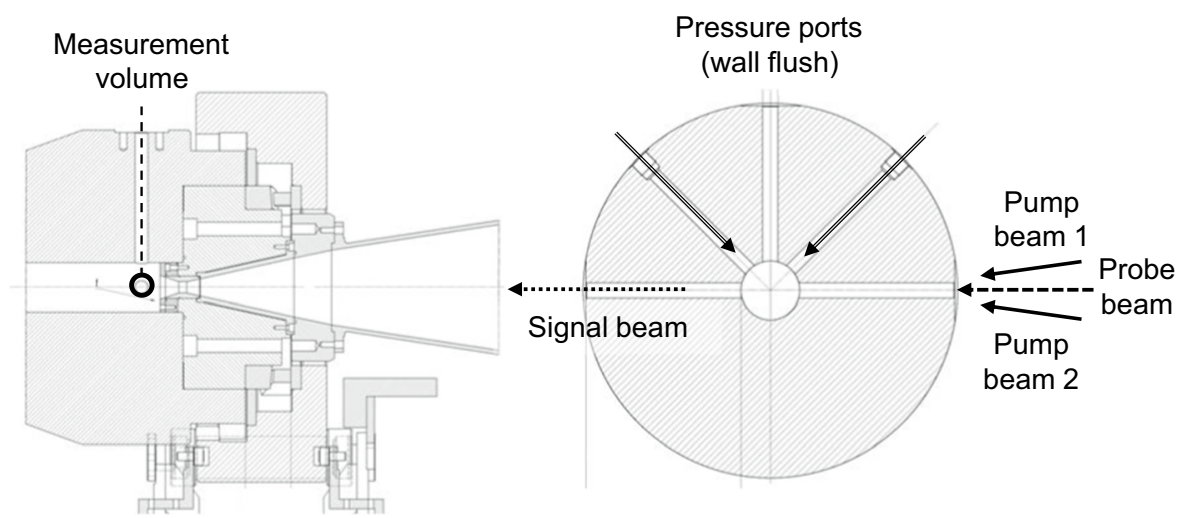




\subsection{Laser optical set-up}

The optical set-up for the generation of resonant acoustic gratings consists of a pulsed frequency-doubled Nd:YAG laser (Innolas SpitLight 2000-10; wavelength $532 \mathrm{~nm}$; max. pulse energy $1000 \mathrm{~mJ}$; pulse duration $7 \mathrm{~ns}$ ) as the pump source and a dye laser (Radiant Dyes NarrowScan; dye Rhodamine B; efficiency $\sim 30 \%$ ) to yield an excitation wavelength range of 590-600 $\mathrm{nm}$. The continuous probe beam is provided by an Ar-ion laser (Coherent Innova 90-4) operating at $514 \mathrm{~nm}$. A $500 \mathrm{MHz}$ photomultiplier tube (TSI) is employed for signal detection, and a digital storage oscilloscope (LeCroy) with $1 \mathrm{GHz}$ bandwidth serves for discretization at $10 \mathrm{GS} / \mathrm{s}$. For the measurements presented herein, the power of the irradiated probe beam is $250 \mathrm{~mW}$ and the pulse energy of the pump beams measures $40 \mathrm{~mJ}(20 \mathrm{~mJ}$ each). The latter is deliberately kept as low as possible such as to not approach the damage threshold of the optical sapphire windows, yet to yield sufficient amplitudes for thermal (resonant) LIGS measurements.

In general, the variation of excitation wavelength allows for separate as well as simultaneous excitation of resonant and non-resonant gratings. In the present investigation, due to the absence of an adequate electronic transition in air, pure resonant gratings are generated by resonant excitation of nitric dioxide $\left(\mathrm{NO}_{2}\right)$ at $\sim 593 \mathrm{~nm}$. The relevant absorption system is a broadband electronic transition of $\mathrm{NO}_{2}$, ranging from the ultraviolet spectrum $\left(\mathrm{B}^{2} \mathrm{~B}_{2}-\mathrm{X}^{2} \mathrm{~A}_{1}\right) \sim 250 \mathrm{~nm}$ to the visible $\left(A^{2} B_{1}-X^{2} A_{1}\right) \sim 650 \mathrm{~nm}$, exhibiting a global maximum at $435 \mathrm{~nm}[35,36]$. Due to high overlap of several separate transitions in the wavelength range considered, the absorbance of $\mathrm{NO}_{2}$ is expected to not decrease noticeably for higher temperatures as in the case of continued depletion of ground states. Particularly at elevated pressures, as present in shock tunnels, this is considered favourable with regard to pressure line broadening which can be an impairment when exploiting single-line absorption features as in the case of many diatomic molecules. Hence, the broadband absorbance of $\mathrm{NO}_{2}$ will foreseeably not pose an obstacle for LIGS application under high-enthalpy, high-temperature conditions.

While the general set-up is similar to the optical set-up of a prior small-scale shock tube study by the authors [19], significantly more effort had to be invested in the laser light guidance and optical pathway, as optical access to the HELM shock tunnel is merely provided via small-diameter radial bores, rather than large surface area windows, see Fig. 4. Here, in addition to a geometrically closely confined laser beam pathway into and out of the measurement volume located at the shock tube centerline, problems arose due to the inevitable recoil of the high-enthalpy free-piston impulse facility [20]. Upon transient operation, compression piston acceleration and deceleration cause a considerable axial displacement of about $30 \mathrm{~mm}$ of the entire shock tunnel which had to be accounted for in order to ensure pump, interrogation, and signal laser beams to accurately pass through the optical access ports.

Therefore, laser beams are to be guided in parallel to the shock tube as far as possible. While laser equipment and optical components are situated adjacent to and hence mechanically decoupled from the facility, only the deflecting mirrors and focusing lenses-imperative for accurate laser beam guidance into/out of the test volume as well as focusing beams to intersect at the centerline-are attached to the shock tube itself. By being guided alongside in axial direction, it is ensured that laser beams are not deflected away from the radial bores and that they pass through the focusing lenses despite facility recoil throughout the experiment. The focal lengths of the focusing lenses are set to $500 \mathrm{~mm}$, to yield a geometrical crossing full angle $\Theta$ of $\sim 1.17 \mathrm{deg}$. To achieve an improved signal-to-noise ratio (SNR) by geometrical and optical transmission of the diffracted probe, i.e., signal, beam only, spatial pinhole and optical filters are inserted into the detection pathway; see Fig. 5.

Earlier LIGS measurements by the authors $[20,21]$ were affected by problems related to optical beam alignment and facility displacement due to transient mechanical recoil. The success rate of optical measurements was compromised by recessed mounting of optical sapphire windows to the ST outer diameter, rather than the inner diameter, see Fig. 4. Accordingly, high-aspect-ratio $(L / d \sim 10)$ geometric cavities resulting from radial access bores within the nozzle reservoir section demanded laser beams to travel over large radial distance of $0.6 \mathrm{~m}$ through the stagnating test gas close to the endwall. In these cases, it became evident that the mandatory geometrical alignment of irradiated pump/probe beam and coherent signal beam was significantly disturbed by strong secondary flow and gradual deflection, as entailed by transient compression and expansion waves, pronounced density gradients, and vortical motion. This resulted in a significantly limited success rate of single-shot measurements by LIGS as a result of strong beam steering and displacement of the coherent signal beam off the detecting PMT and pinhole apertures [20]. Recently, the opto-mechanical set-up, i.e., coupling between optical laser components and HELM facility, has been revised extensively such that optical sapphire windows are now flush-mounted with respect to the ST inner diameter. Thereby, radial cavities within the nozzle reservoir are avoided, thus minimizing vortical motion and radial density gradients towards the endwall region as a whole and effectively reducing the distance over which all four laser beams are to traverse the perturbed test medium. Furthermore, a hydraulic damping system based on heavyduty shock absorbers has been installed recently which guide reaction forces into the foundation and thus effectively limit strong axial displacement of the facility. 
Fig. 5 Schematic of the LIGS optical set-up [19]

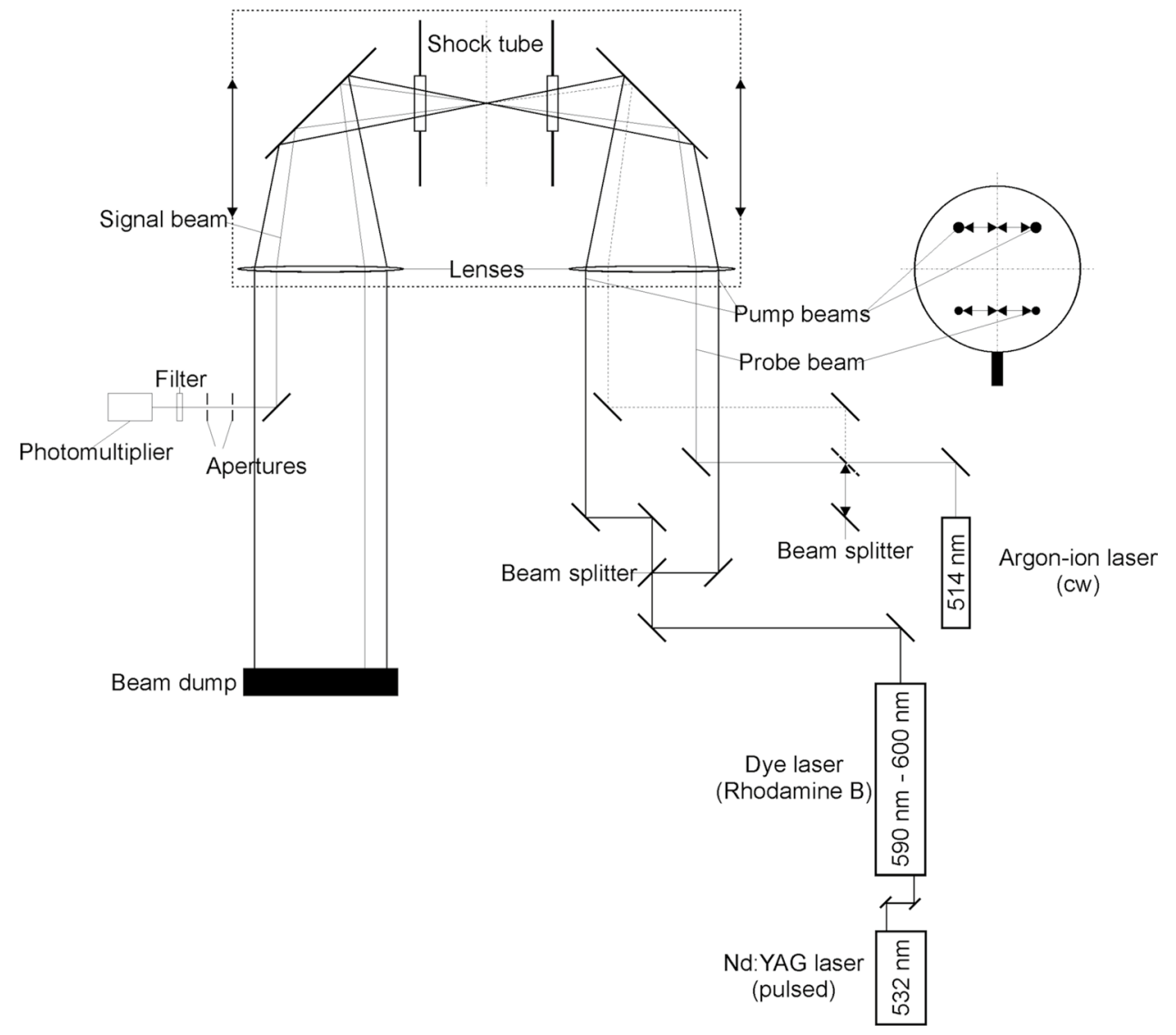

A significantly enhanced beam path stability, i.e., robustness of beam alignment, resulted as direct improvement of these measures which now further allows better rejection of spurious reflections and stray light by smaller geometrical pinhole apertures. Accordingly, the ensuing success rate of single-shot LIGS measurements presented herein could be effectively increased to near $90 \%$. As a side effect, inserting flush-mounted windows results in a smaller pump beam intersection angle $\Theta$ which entails an increased fringe spacing constant $\Lambda$. However, this proves advantageous due to longer signal lifetimes, resulting from an increased number of signal oscillations, i.e., induced sound wave periods, effectively increasing measurement accuracy, particularly when frequency-domain DFT is used for signal processing [37].

\section{Experimental methodology}

\subsection{Technical approach}

In the experiments reported herein, time-accurate triggering of a single pump laser pulse is provided by means of the response of the PCB pressure transducer mounted in the measurement plane, when the incident shock transits.
A delay generator ensures the laser pulse to pass the measurement volume shortly after reflected shock wave passage. While the pump laser operates at a fixed repetition rate of $10 \mathrm{~Hz}$, a manufacturer-added feature allows firing an additional intermediate laser pulse that is necessary for timeaccurate response to the shock passing event. A manual time delay of $235 \mu$ s between single gating of the Nd:YAG laser flash lamp and Q-switch is deliberately set to allow for maximum pump laser beam energy emission, see [19] for details. Jitter on the order of $\sim \mathrm{O}(10 \mathrm{ps})$ is found to be insignificant for the instant of pulse emission.

As direct (geometrical) measurement of the optical setup's pump laser beam intersection angle $\Theta$ is unfeasible and prone to error, a unique reference calibration is conducted at ambient temperature and shock tube initial fill pressure (state 1), prior to every single experiment. This is to ensure unambiguous determination of the optical grating constant (interference fringe spacing) $\Lambda$ from acquired calibration signal oscillation frequency. For means of consistency, reference point calibration employs the identical real gas (equation of state) thermophysical reference data according to Lemmon et al. [38], to uniquely determine air speed of sound at reference pressure and temperature, based on reference thermocouple measurement. Having determined calibration point oscillation frequency and reference speed of sound, the exact 
grating constant $\Lambda$ is thus calculated from (3). Throughout the experiment, $\Lambda$ is henceforth used to derive test gas speed of sound from the oscillation frequency acquired under test conditions (state 5). Analogously, corresponding test gas temperature $T_{5}(\exp )$ is calculated by a backward interpolation of the thermophysical reference data in [38], based on measured pressure and sound speed.

Homodyne detection temperature measurements by LIGS are conducted by detection of the diffracted signal beam and subsequent frequency-domain analysis. The typical approach for determination of the signal scattering frequency $f_{\mathrm{M}}$ found in the literature is the discrete fast Fourier transform (DFT). A comprehensive approach is the numerical fitting of the theoretical signal temporal evolution, as postulated by Cummings et al. [26], to the experimentally acquired time-domain signal via a nonlinear least-squares fit. Apart from speed of sound - and thus temperature-further thermophysical properties of the fluid such as thermal diffusivity and acoustic damping rate can thus be extracted from the temporal decay rate of the signal envelope [29]. Performance of both data reduction procedures is limited by the acquired time-domain signal quality, in terms of signal length, i.e., oscillation lifetime, as well as the presence of background noise distortion [20]. In the current work, both time- and frequency-domain procedures are performed separately for distinct signal analysis. Based on incident shock Mach number $M_{\mathrm{s}}$ derived from time-of-arrival pressure gauges, postreflected shock pressure $p_{5}(\exp )$ and post-reflected shock reference temperatures $T_{5}($ th $)$ are calculated either by NASA's CEA code [39] or via ideal gas 1-D shock jump relations.

So far, the overall highest test conditions at which quantitative measurements of translational temperature by LIGS in a reflected shock tunnel have been made are $1100 \mathrm{~K}$ and $6 \mathrm{MPa}$ stagnation temperature and pressure, respectively, behind an incident shock wave of $M_{\mathrm{s}}=2.6$ in air [20]. In order to demonstrate measurement technique feasibility under increasingly severe conditions and in a less benign environment, the test conditions in the full-scale highenthalpy facility HELM are successively increased to $1900 \mathrm{~K}$ and $22 \mathrm{MPa}$. Here, proposing LIGS as a laser diagnostics technique suitable for in situ determination of stagnation conditions in ground test facilities, single-shot resonant LIGS is applied for translational temperature measurement at three low-medium-enthalpy test conditions (case C1-C3) in the nozzle reservoir of the HELM shock tunnel, assuming thermo-chemical equilibrium. Initial facility operation parameters are presented in Table 1. Three different test cases have been set by varying the shock tube pressure (state 1) from 0.15 to $0.5 \mathrm{MPa}$ for a fixed diaphragm burst pressure of $22 \mathrm{MPa}$; all experiments are conducted in dry air, as driver and test gas in the CT and ST, respectively. In the case of all 20 experiments, the HELM facility is operated as a shock tube with closed endwall.

Post-reflected shock (state 5) stagnation pressure, temperature, and enthalpy of up to $22.5 \mathrm{MPa}, 1650 \mathrm{~K}$, and $2.2 \mathrm{MJ} /$ $\mathrm{kg}$, respectively, within the nozzle reservoir are detailed in Table 1, based on the measured incident shock Mach number. Nozzle reservoir conditions throughout the experiment are predicted by quasi-1-D CFD simulation, using Jacobs' L1d code [40] and NASA's Gordon McBride CEA code for the calculation of equilibrium thermophysical properties [39]. L1d performance for time-resolved simulation of shortduration test facilities with free-piston driver configurations is well documented due to validation in reflected shock tunnel and expansion tube facilities [41].

\subsection{Calibration procedure}

As post-shock temperatures and thus intrinsic formation of nitric dioxide due to shock heating are still considered low, NO is seeded as tracer gas (235-500 ppm) in the shock tube section in order to ensure formation of $\mathrm{NO}_{2}$ (via chemical equilibrium at room temperature) as active species for resonant (thermal) grating excitation at $593 \mathrm{~nm}$. Based on $\mathrm{NO}_{2}$ seeding concentration and low pump beam pulse energies of $20 \mathrm{~mJ}$, contributions of an electrostrictive (non-resonant) grating component-physically present at very low intensity - are entirely negligible (at no time present, i.e., noticeable, neither in signal time nor in frequency domain) in comparison with the intensity of the deliberately enforced, dominant thermal (resonant) grating exploited throughout both calibration and measurements - compare [23]. In order to ensure homogeneous species distribution, a seeding gas mixture (5000 ppm NO diluted in $\mathrm{N}_{2}$ ) is inserted into the shock tube just after evacuation and before setting the ST initial fill pressure $p_{1}$ by inserting dry air.

Table 1 Test case conditions: shock tube and rupture pressure levels and nominal reservoir stagnation conditions (state 5) as predicted by CEA

\begin{tabular}{lllllllll}
\hline Test case & \multicolumn{2}{l}{ Facility pressures } & Incident shock & \multicolumn{2}{l}{ Nozzle reservoir $($ state 5) } \\
\cline { 2 - 3 } \cline { 6 - 8 } & ST $(\mathrm{MPa})$ & Rupture $(\mathrm{MPa})$ & $M_{\mathrm{s}}(-)$ & & $p_{5}(\mathrm{MPa})$ & $T_{5}(\mathrm{~K})$ & $h_{5}(\mathrm{MJ} / \mathrm{kg})$ & $\rho_{5}\left(\mathrm{~kg} / \mathrm{m}^{3}\right)$ \\
\hline $\mathrm{C} 1$ & 0.5 & 22 & 2.85 & 22.5 & 1200 & 1.4 & 6.51 \\
$\mathrm{C} 2$ & 0.25 & 22 & 3.10 & 14.5 & 1360 & 1.5 & 3.69 \\
$\mathrm{C} 3$ & 0.15 & 22 & 3.50 & 12.0 & 1650 & 1.9 & 2.53 \\
\hline
\end{tabular}

Enthalpy is referenced to $0 \mathrm{~K}$ 
Influence of seeding concentration (maximum $0.05 \mathrm{vol} \%$ ) on specific gas constant $R_{\mathrm{S}}$ is taken into account. For every experiment presented herein, a separate baseline reference calibration of the optical grating is carried out a priori and in situ, immediately before facility operation. To ensure calibration to be most representative of the following test case experiment, (state 1) shock tube initial conditions of the quiescent test gas are set as reference, such that, e.g., grating calibration for case $\mathrm{C} 2$ is carried out at 2.5 bar shock tube initial fill pressure, with $235 \mathrm{ppm}$ NO seeding. Every single calibration involves a set of 200 single-shot LIGS signals, acquired at $10 \mathrm{~Hz}$ pump laser repetition rate, to yield a single, 200-fold averaged calibration curve for accurate determination of reference condition oscillation frequency (fringe spacing). Local reference gas temperature is measured via an adjacent thermocouple with $0.1 \mathrm{~K}$ precision, to yield air speed of sound at reference condition. For means of consistency with data analysis at test conditions, real gas (equation of state) values according to Lemmon et al. [38] are similarly utilized for calibration purposes. Henceforth, the interference fringe spacing (optical grating constant) $\Lambda$ is uniquely determined according to (3).

Throughout $20 \mathrm{~s}$ of calibration time duration, fringe spacing and oscillation frequency reflect a normal distribution with nominal values of $\mu_{\lambda}=14.55 \mu \mathrm{m}$ and $23.690 \mathrm{MHz}$, respectively. An absolute standard deviation $\sigma_{\lambda}=0.021 \mu \mathrm{m}$ results in a relative standard deviation for the grating constant of $\sigma_{\lambda} / \mu_{\lambda}=0.20 \%$, reflecting high precision and robustness of a priori optical set-up calibration. Employing $10 \mathrm{GHz}$
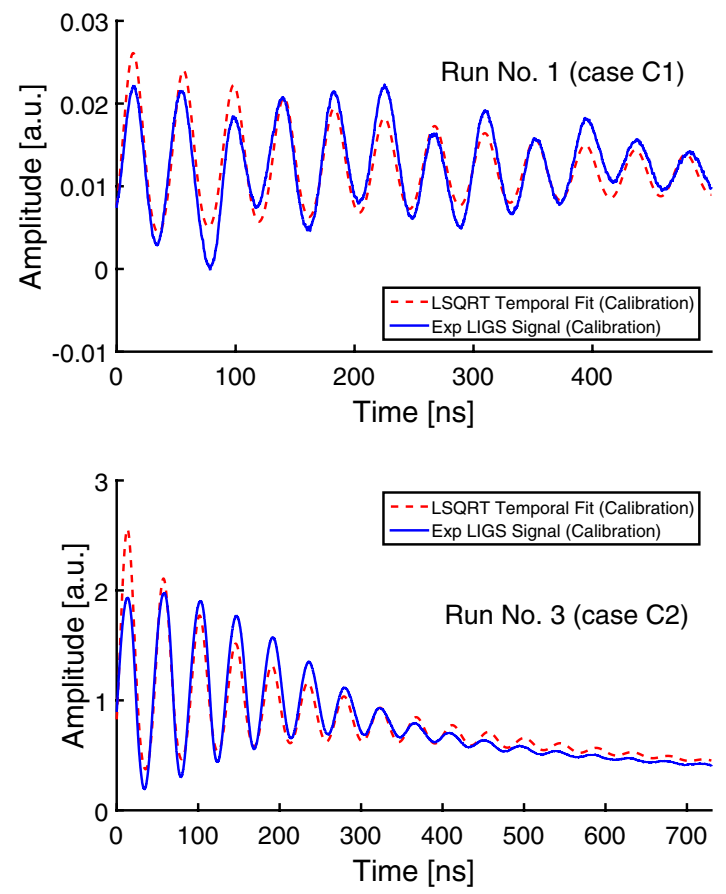

sampling frequency and deriving the reference calibration curve from a 200 -fold waveform average, reference frequency, i.e., fringe spacing, calibration precision is deduced to be even lower, as discussed by Balla and Miller [19]. As two competitive data reduction methodologies are being used for time- and frequency-domain analysis of single-shot LIGS signals to be acquired at experimental test condition, reference condition calibration is likewise conducted by separate application of signal time evolution fitting and DFT; this is to ensure signal analysis for both methodologies to be distinct. A background subtraction routine is used for both techniques to minimize stray light noise influence. With a relevant laser pulse length of $\sim 10 \mathrm{~ns}$, turbulence and convection effects on the opto-acoustic grating (of sub-microsecond duration $\sim 100 \mathrm{~ns}$ ) are not considered relevant and hence are not treated separately in the data analysis [27, 37].

Individual LIGS calibration curves of four representative test runs for cases $\mathrm{C} 1-\mathrm{C} 3$ are presented in Fig. 6, conducted at room temperature and immediately before the experiment. Each curve presents a 200-fold average of successive single shots. While calibration signal oscillation at reference conditions is seen to last over a time duration of approximately $1 \mu \mathrm{s}$ and at later time instants gradually decreases due to thermal conduction and acoustic damping [42], only the initial closely correlated signal of the oscillation is used for calibration purposes. As the concentration of $\mathrm{NO}_{2}$ as resonantly excited species in dry air is too low to obtain a proper calibration signal at room temperature, test cases $\mathrm{C} 1-\mathrm{C} 3$ have a seeding concentration of NO between 235
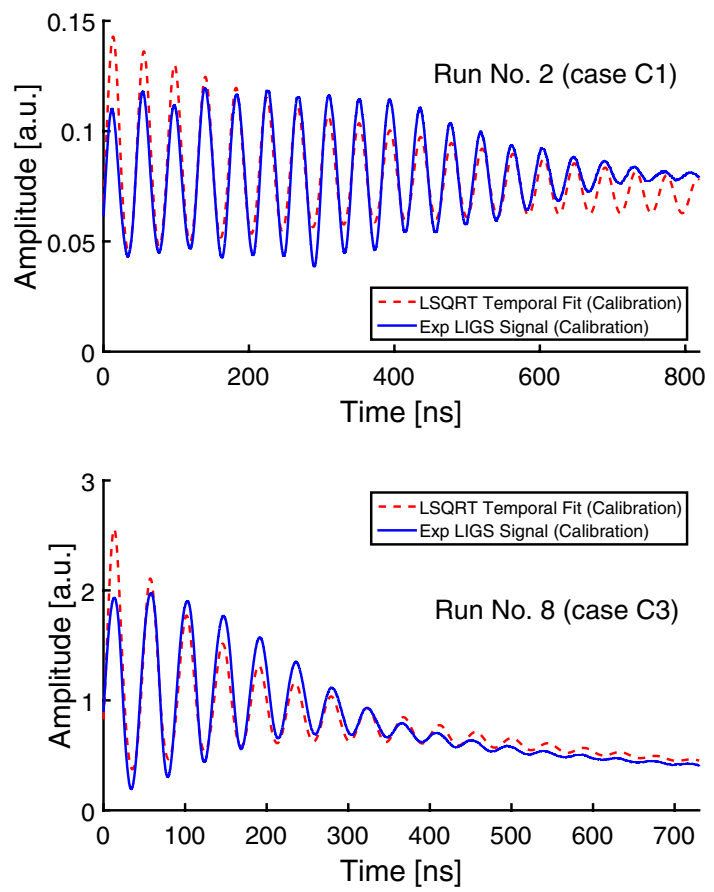

Fig. 6 Time-domain LIGS calibration signals prior to runs C1-C3 with temporal curve fit 
and $500 \mathrm{ppm}$, depending on the initial ST pressure. Seeding induces greater (resonant) signal intensity and thus favourably affects the length of the useful calibration curve, varying between 500 and $800 \mathrm{~ns}$. This has, however, no influence on measurement accuracy, as different seeding concentrations are taken into account.

In Fig. 6, besides the experimentally determined calibration curve, a numerical least-squares fit to the signal's temporal evolution is plotted, illustrating excellent qualitative and quantitative agreement of the oscillation crests and troughs as well as its damped oscillation envelope. Depending on room temperature and beam path, the calibration signal scattering frequency for individual experiments is determined to vary between 22.546 and $23.673 \mathrm{MHz}$, corresponding to a spatial grating constant $\Lambda$ of $15.253-14.556 \mu \mathrm{m}$. Accordingly, the pump beam crossing angle $\Theta$ varied between $1.1^{\circ}$ and $1.16^{\circ}$.

\subsection{Uncertainty analysis}

Measurement uncertainty is estimated according to the single sample method proposed by Kline and McClintock [43]. Ambient air reference temperature is read with a thermocouple precision of $0.1 \mathrm{~K}$, such that the absolute uncertainty of baseline calibration test gas temperature is estimated to be at most $0.33 \%$ ( $\pm 1 \mathrm{~K}$ at $300 \mathrm{~K}$ room temperature). Henceforth, linear interpolation of high-temperature and high-pressure thermophysical reference data for dry air, provided in Lemmon et al. [38], is employed to obtain air speed of sound from measured thermocouple temperature. Based on $0.2 \%$ tabulated uncertainty for air speed of sound, as reported in [38], calibration uncertainties of the LIGS grating constant $\Lambda$, intersection angle $\Theta$, and Bragg angle $\varphi$ are estimated to be $1.07 \%$. Herein, the major contributor to grating calibration uncertainty is the DFT-inherent frequency discretization, which yields deviations below $1.0 \%$.

Employing the calibrated grating constant to determine air speed of sound from measured oscillation frequency and henceforth calculating gas temperature, absolute measurement uncertainty of the latter is inferred to amount to $\pm 55 \mathrm{~K}$ and $\pm 50 \mathrm{~K}$ for the DFT and curve fit, respectively. On a relative scale, uncertainty of post-reflected shock wave test gas temperature $T_{5}(\exp )$ amounts to maximum $5.0 \%$ and $4.6 \%$ when compared to the lowest nominal temperature (1100 K) of test case $\mathrm{C} 1$. For higher temperature test conditions $\mathrm{C} 2$ and $\mathrm{C} 3$, the relative measurement error will hence be notably lower than $4.0 \%$.

Similarly, as theoretical reference temperatures are calculated by CEA, based on the experimentally acquired incident shock velocity, measurement uncertainties in shock speed, i.e., Mach number, directly translate to reference temperature variation. Here, Mach number uncertainty is dictated by the data acquisition system's temporal resolution and deviations from the nominal relative distance of PCB wall pressure gauges, which amounts to a maximum error of $0.91 \%$ in Mach number. Accordingly, measurement uncertainty in associated post-reflected shock (state 5 ) reference temperature $T_{5}(\mathrm{th})$ and pressure $p_{5}(\mathrm{th})$ is estimated to be $1.5 \%$ and $2.7 \%$, respectively. Measurement uncertainty in experimental pressure $p_{5}(\exp )$ is negligible, considering a discretization error of merely $0.02 \%$, as given by the 14-bit amplitude resolution of the transient recorder.

\section{Results}

\subsection{Experimental test condition}

In order to demonstrate the applicability of LIGS in the real test environment of a full-range reflected shock tunnel facility, 20 successful measurements for three (case C1-C3) low-medium-enthalpy test conditions $(1.7-2.2 \mathrm{MJ} / \mathrm{kg})$ at stagnation pressures and temperatures up to $22 \mathrm{MPa}$ and $1900 \mathrm{~K}$, respectively, are presented in Table 2. Here, the Mach number of the incident shock wave $M_{\mathrm{s}}(\exp )$ (based on time-of-arrival measurements in the vicinity of the reflecting ST endwall) is detailed for all 20 experiments and the measured post-reflected shock stagnation pressure $p_{5}(\exp )$ is compared to the corresponding CEA prediction $p_{5}($ th $)$, based on incident shock Mach number and assuming thermo-chemical equilibrium, see Table 2.

Measurement volume pressure traces within the nozzle reservoir for representative test runs of conditions C1-C3 are shown in Fig. 7, plotted on a relative time axis. In addition, CEA-predicted post-shock pressures $p_{5}($ th) for each case are illustrated by solid horizontal lines. By varying the ST pressure of $0.5 \mathrm{MPa}, 0.25 \mathrm{MPa}$, and $0.15 \mathrm{MPa}(\mathrm{C} 1, \mathrm{C} 2$, and $\mathrm{C} 3$ ), the condition $\mathrm{C} 1$ is seen to be strongly undertailored, reflecting a post-shock pressure $p_{5}(\exp )$ that steadily decreases due to the expansion fan originating from contact surface interaction with the endwall-reflected shock wave. In contrast, varying the ST pressure to $0.25 \mathrm{MPa}$ and $0.15 \mathrm{MPa}$ $(\mathrm{C} 2, \mathrm{C} 3)$ results in an overtailored, i.e., initially near-tailored, pressure profile with stagnation pressure for $\mathrm{C} 2$ and C3 remaining nearly constant around 15.5 and $13.6 \mathrm{MPa}$, respectively, for a limited duration of $0.7 \mathrm{~ms}$ after reflected shock passage. Pressure at the laser pulse time instant upon Q-switch gating (as indicated by the vertical dashed lines) is observed to yield a close approximation of the time-mean post-reflected shock wave pressure $p_{5}(\exp )$ for all 20 test cases. Measured pressure $p_{5}(\exp )$ is found to be generally lower than reference pressure as predicted by CEA. Deviations of around $-10 \%$ are in good agreement with expected pressure levels, attributed to losses that are not taken into account in the 1-D inviscid calculations by CEA. Further, gradual attenuation of the incident shock wave in the ST, 
Table 2 Summary of experimental parameters per test run: incident shock Mach number and stagnation pressure

\begin{tabular}{|c|c|c|c|c|c|c|c|c|c|c|c|}
\hline \multirow[t]{2}{*}{ Run no. } & \multirow[t]{2}{*}{ Test case } & \multirow{2}{*}{$\begin{array}{l}\operatorname{Exp} \\
M_{\mathrm{s}}(-)\end{array}$} & \multirow{2}{*}{$\begin{array}{l}\operatorname{Exp} \\
p_{5}(\mathrm{MPa})\end{array}$} & \multirow{2}{*}{$\begin{array}{l}\text { Th }(\mathrm{CEA}) \\
p_{5}(\mathrm{MPa})\end{array}$} & \multirow{2}{*}{$\begin{array}{l}\operatorname{Exp}(\text { Fit }) \\
T_{5}(\mathrm{~K})\end{array}$} & \multicolumn{3}{|c|}{ Th (CEA) } & \multicolumn{3}{|c|}{ Th (1-D inviscid) } \\
\hline & & & & & & $T_{5}(\mathrm{~K})$ & $\Delta T(\mathrm{~K})$ & $\Delta T(\%)$ & $T_{5}(\mathrm{~K})$ & $\Delta T(\mathrm{~K})$ & $\Delta T(\%)$ \\
\hline 1 & $\mathrm{C} 1$ & 2.678 & 21.40 & 16.52 & 1098 & 1089 & 9 & 0.8 & 1151 & -53 & -4.6 \\
\hline 2 & $\mathrm{C} 1$ & 2.855 & 21.87 & 19.86 & 1115 & 1120 & -5 & -0.5 & 1278 & -163 & -12.8 \\
\hline 3 & $\mathrm{C} 2$ & 3.114 & 15.17 & 14.52 & 1548 & 1360 & 188 & 13.8 & 1478 & 70 & 4.7 \\
\hline 4 & $\mathrm{C} 2$ & 3.173 & 13.78 & 15.33 & 1737 & 1398 & 339 & 24.3 & 1528 & 209 & 13.7 \\
\hline 5 & $\mathrm{C} 2$ & 2.958 & 16.15 & 12.53 & 1303 & 1267 & 36 & 2.8 & 1367 & -64 & -4.7 \\
\hline 6 & $\mathrm{C} 2$ & 2.982 & 17.40 & 12.85 & 1313 & 1282 & 31 & 2.4 & 1386 & -73 & -5.3 \\
\hline 7 & $\mathrm{C} 2$ & 2.990 & 14.72 & 12.96 & 1320 & 1292 & 28 & 2.2 & 1398 & -78 & -5.6 \\
\hline 8 & $\mathrm{C} 3$ & 3.569 & 11.23 & 12.75 & 1775 & 1676 & 99 & 5.9 & 1877 & -102 & -5.4 \\
\hline 9 & C3 & 3.186 & 13.62 & 9.30 & 1350 & 1412 & -62 & -4.4 & 1544 & -194 & -12.6 \\
\hline 10 & $\mathrm{C} 3$ & 3.271 & 9.48 & 10.03 & 1361 & 1471 & -110 & -7.5 & 1618 & -257 & -15.9 \\
\hline 11 & $\mathrm{C} 3$ & 3.488 & 10.71 & 11.98 & 1694 & 1624 & 70 & 4.3 & 1812 & -118 & -6.5 \\
\hline 12 & $\mathrm{C} 3$ & 3.596 & 11.69 & 13.03 & 1887 & 1703 & 184 & 10.8 & 1913 & -26 & -1.4 \\
\hline 13 & $\mathrm{C} 3$ & 3.573 & 11.43 & 12.78 & 2231 & 1691 & 540 & 31.9 & 1900 & 331 & 17.4 \\
\hline 14 & $\mathrm{C} 3$ & 3.589 & 11.30 & 12.96 & 1914 & 1705 & 209 & 12.3 & 1917 & -3 & -0.2 \\
\hline 15 & $\mathrm{C} 3$ & 3.498 & 10.63 & 12.05 & 1916 & 1638 & 278 & 17.0 & 1831 & 85 & 4.6 \\
\hline 16 & $\mathrm{C} 3$ & 3.545 & 11.13 & 12.51 & 1834 & 1676 & 158 & 9.4 & 1880 & -46 & -2.5 \\
\hline 17 & $\mathrm{C} 3$ & 3.484 & 10.64 & 11.93 & 1857 & 1631 & 226 & 13.9 & 1822 & 35 & 1.9 \\
\hline 18 & $\mathrm{C} 3$ & 3.332 & 9.97 & 10.54 & 1692 & 1525 & 167 & 11.0 & 1686 & 6 & 0.4 \\
\hline 19 & C3 & 3.543 & 10.28 & 11.67 & 1660 & 1614 & 46 & 2.9 & 1799 & -139 & -7.7 \\
\hline 20 & $\mathrm{C} 3$ & 3.498 & 10.75 & 12.10 & 1771 & 1650 & 121 & 7.3 & 1845 & -74 & -4.0 \\
\hline
\end{tabular}

Results of temperature measurements: LIGS single-shot temperature and theoretical reference temperatures, including absolute and relative deviations, behind the reflected shock (state 5)

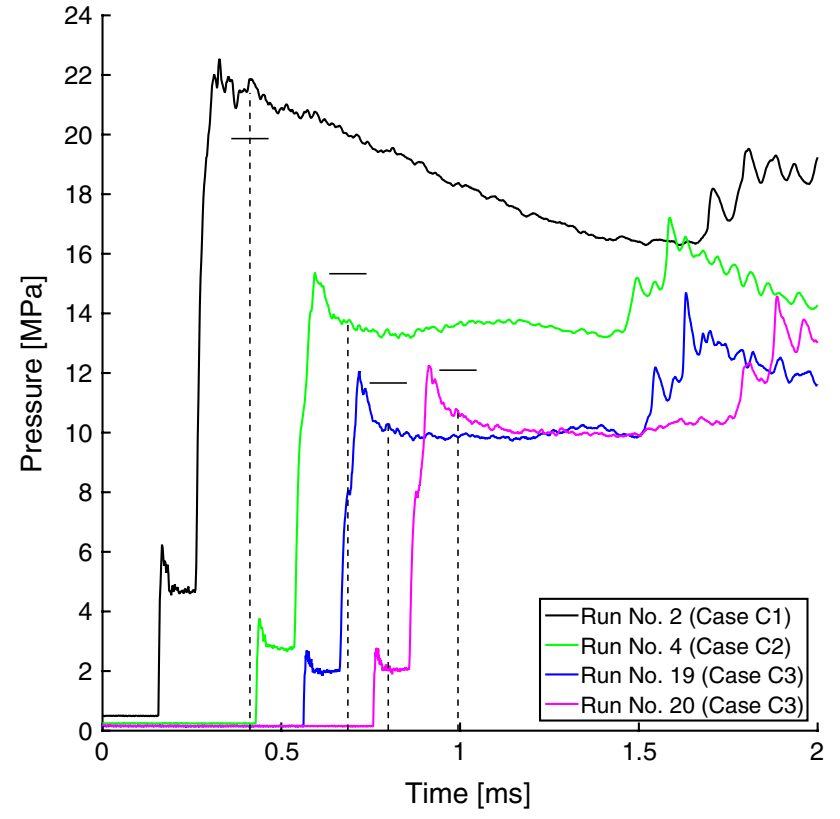

Fig. 7 Experimental pressure traces in the nozzle reservoir for runs C1-C3. Time instant of Q-switch trigger behind the reflected shock wave marked by dashed line measured around $390 \mathrm{~mm}$ upstream of the ST endwall, may occur. However, for some measurements, as is observed in Fig. 7, the pressure $p_{5}(\exp )$ is found to be $10.1 \%$ higher than $p_{5}($ th) for test run 2. Even though the precise cause of this underestimation of pressure by CEA at the overall lowest incident shock Mach number of 2.678 remains yet unclear, it is assumed to be attributed to the evident undertailored pressure trend of condition C1. Precisely, as CEA is a 1-D code absent of viscous effects and time-resolved calculation of post-reflected shock quantities, this post-shock temporal variation, i.e., decay, of state quantities is not captured by the code. This is in contrast to conditions $\mathrm{C} 2$ and $\mathrm{C} 3$ which are considered near-tailored, i.e., quasi-steady, for the relevant test time and where measured pressures are in good agreement with CEA predictions. Thus, the quantitative experimental pressure trace $p_{5}(\exp )$ for test run 2 (case $\mathrm{C} 1$ ) is considered accurate.

\subsection{LIGS temperature measurement}

Q-switch gating and pump laser pulse release throughout the experiment are synchronized to the incident shock passage event, triggered by the rising flank of the pressure gauge coincident with the measurement location. Manual delay is set to $235 \mu$ s to gear gating of the Q-switch to a suitable time 
instant shortly after reflected shock wave passage. Timedomain resonant LIGS signals obtained from single-pulse measurements are presented in Fig. 8 for four representative test runs of cases C1-C3. Here, for means of illustration of robustness of the temporal least-squares fit post-processing procedure, signal curves are selectively subjected to moving-average (Savitzky-Golay) filters of varying step width. While the temporal signal of run 3 remains unfiltered, noise level is gradually reduced for runs 1,2 , and 8 , respectively. Whereas a priori filtering of the raw signal before signal analysis can be advantageous for DFT frequency analysis, the time-domain nonlinear least-squares curve fitting procedure is found to be very robust against background noise and to be entirely unaffected by signal filtering. However, for initial visual identification of relevant signal length, minor filtering can be beneficial. Here, temporal duration of the wellcorrelated single-shot signal is seen to vary between 100 and $300 \mathrm{~ns}$ for cases $\mathrm{C} 1-\mathrm{C} 3$, respectively. Within this time period, a dominant and harmonic signal oscillation of a single (discrete) frequency is clearly observed, superimposed by a low level of small-amplitude distortions and spurious background noise. As expected for laser-induced gratings, signal beam oscillation amplitude (envelope) decreases with time, as density grating lifetime is limited by thermal diffusion and acoustic damping. For later time instances, signal oscillation gradually diminishes and cannot be further separated from background noise. For all four runs, the nonlinear curve fit is seen to closely coincide with the experimental signal curve where the dominant oscillation frequency is accurately captured. Moreover, besides signal scattering frequency, signal amplitudes are seen to be well matched by the fit and the gradual amplitude decay to be captured in the beginning as well as towards the end of the signal time period. Furthermore, signal amplitudes of oscillation crests and troughs are observed to be similarly well captured, even at later time instances where the signal gradually decreases to background noise. Overall, the signal with the longest well-correlated lifetime of $300 \mathrm{~ns}$ is found to be captured for run 1 (C1), i.e., at near overall highest pressure and lowest temperature, whereas the shortest signal lifetime is observed for run $8(\mathrm{C} 3)$, reflecting lower pressure and higher temperature. Prevalent background noise was determined in the $\sim 1 \mathrm{GHz}$ range, whereas signal oscillations are detected around $\sim 50 \mathrm{MHz}$. Together with a superior SNR $>20$, this facilitated unambiguous determination of the dominant scattering frequency for every experiment.

In direct comparison with prior measurements in the HELM reservoir at $6 \mathrm{MPa}$ and $1100 \mathrm{~K}$ [20], utilizing the identical optical set-up-yet with a larger pump beam crossing angle $\Theta$ of $3.70^{\circ}$ in comparison with $1.16^{\circ}$ herein-the signal lifetime could be increased by up to $100 \mathrm{~ns}$ which results from a larger extent of the pump beam interference region. This is consistent with geometric considerations, according to which smaller pump beam crossing angles $\Theta$
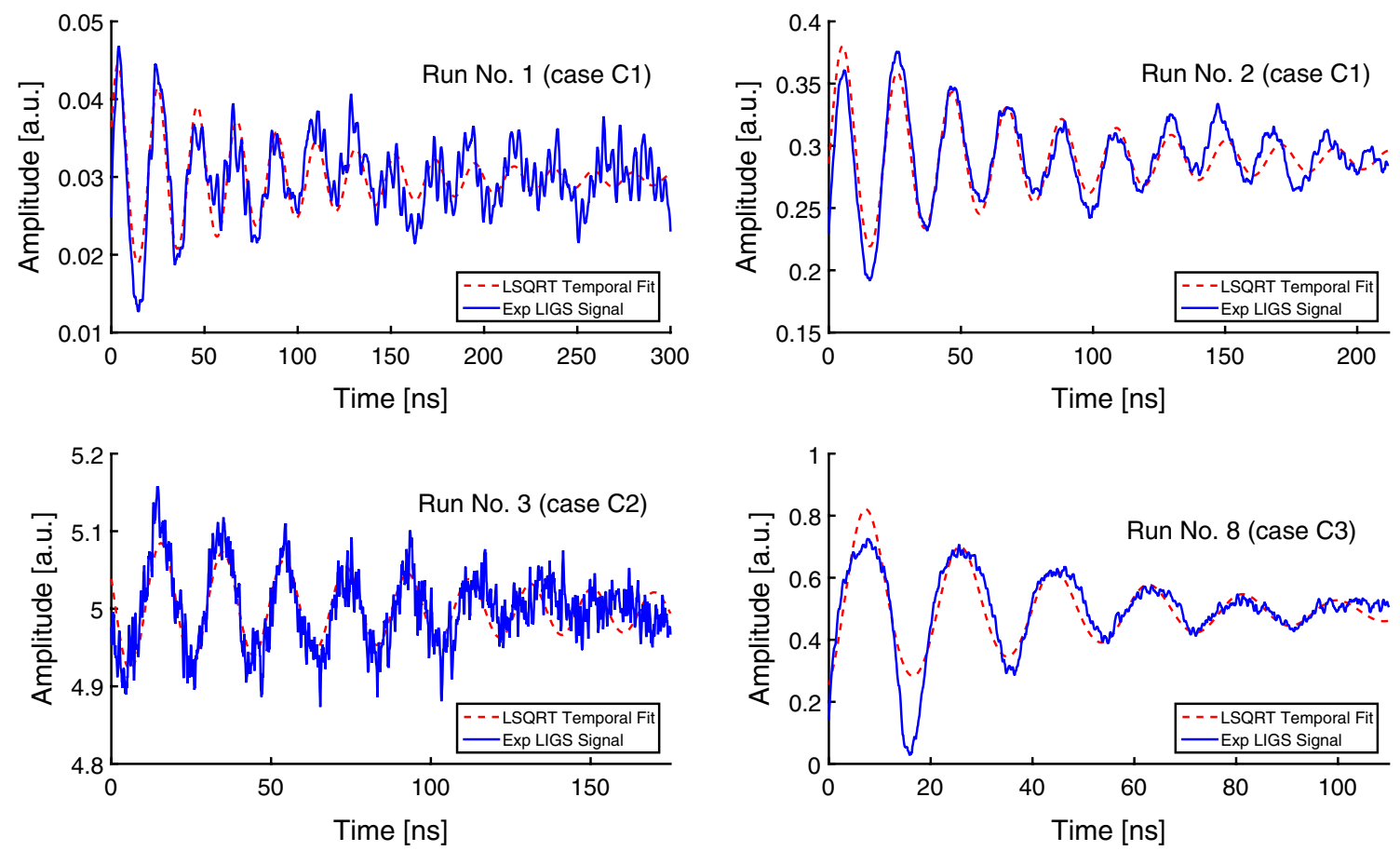

Fig. 8 Time-domain LIGS measurement signals from runs C1-C3 with temporal curve fit. Sorted in ascending order of moving-average filter step width: Run No. 3 , No. 1, No. 2 , No. 8 
induce larger grating constants $\Lambda$, i.e., maximizing the fringe spacing, and hence increase the acoustic period and the number of observable oscillations [37]. Accordingly, rearranging the beam path geometry to smaller intersection angles is favourable with regard to grating lifetime which in general decreases with temperature and thus is the limiting factor for measurement accuracy.

Alternatively to time-domain fitting, LIGS signal oscillations can be processed by a discrete Fourier transformation (DFT) in the frequency domain. Results from both post-processing approaches are in close agreement with one another, reflecting a relative temperature deviation between 0 and $5 \%$. To provide experimental single-shot temperatures with a suitable comparison, theoretical reference temperatures $T_{5}($ th) are calculated by CEA, based on the measured incident shock wave speed, i.e., Mach number $M_{\mathrm{s}}$, acquired by incident shock time-of-arrival gauges, and assuming thermochemical equilibrium. Theoretical post-shock reference temperatures by ESTCj, similarly based on experimental incident shock speed and itself employing CEA for thermochemical reference data, are found to be near identical, yet, generally higher for all 20 individual test runs: reflecting an absolute and a relative deviation from CEA predictions of maximum $5.2 \mathrm{~K}$ or $0.4 \%$, respectively.

\subsection{Stagnation temperature comparison}

For cases $\mathrm{C} 1-\mathrm{C} 3$, measurements are conducted in a temperature range from 1100 to $1900 \mathrm{~K}$, with associated signal scattering frequency and local sound speed ranging from 47.6 to $59.9 \mathrm{MHz}$ and 692 to $863 \mathrm{~m} / \mathrm{s}$, respectively. Compared to theoretical CEA predictions, experimental data evaluated by curve fits yield almost consistently higher oscillation frequency, sound speed, and thus post-shock temperature.
Absolute and relative deviations of experimentally determined temperatures from CEA reference values are detailed in Table 2.

For means of illustration, single-shot and mean temperatures for experimental curve fit (cases C1-C3) as well as reference temperatures according to CEA and 1-D ideal theory are plotted in Fig. 9.

For the complete set of 20 measurements presented, varying agreement between experimental temperatures $T_{5}(\exp )$ and CEA theoretical reference temperatures $T_{5}(\mathrm{th})$ is seen. Precisely, in the case of measurements 1-2 for test condition $\mathrm{C} 1$, remarkably good agreement with a relative deviation below $1 \%$, i.e., below $10 \mathrm{~K}$ absolute deviation, is observed. Moreover, both individual experiments coincide closely, determining post-shock temperature to around $1100 \mathrm{~K}$. This observation is notable, as condition $\mathrm{C} 1$ represents the test case with the overall highest reservoir pressure of $22 \mathrm{MPa}$ and lowest shock Mach number $\sim 2.8$, reflecting a stagnation pressure profile that is clearly undertailored, see Fig. 8.

In the case of condition $\mathrm{C} 2$, the net incident shock Mach number is increased to around 3.1 and the postreflected shock pressure reduced to around $15 \mathrm{MPa}$. For the five test runs (no. 3-7) conducted for case $\mathrm{C} 2$, notably different agreement between experiment and theory is observed. Test runs 3 and 4 yield temperatures $T_{5}(\exp )$ around $1550-1750 \mathrm{~K}$ that are higher than theoretical temperatures $T_{5}(\mathrm{th}) 1360-1400 \mathrm{~K}$, with relative deviation ranging from 13.8 to $24.3 \%$. For experiments $3-4$, the incident shock Mach number of 3.1 was slightly higher than expected, caused by a greater diaphragm burst pressure. As such, with a relative deviation of $24.3 \%$, test run 4 is identified as an outlier. For experiments 5-7, an incident shock Mach number of consistently 3.0 was measured and yielded post-reflected shock temperatures $T_{5}(\exp )$ of $1300-1320 \mathrm{~K}$
Fig. 9 Comparison of LIGS single-shot temperatures behind the reflected shock (state 5). Single points and averages for curve-fit, CEA, and 1-D inviscid theory

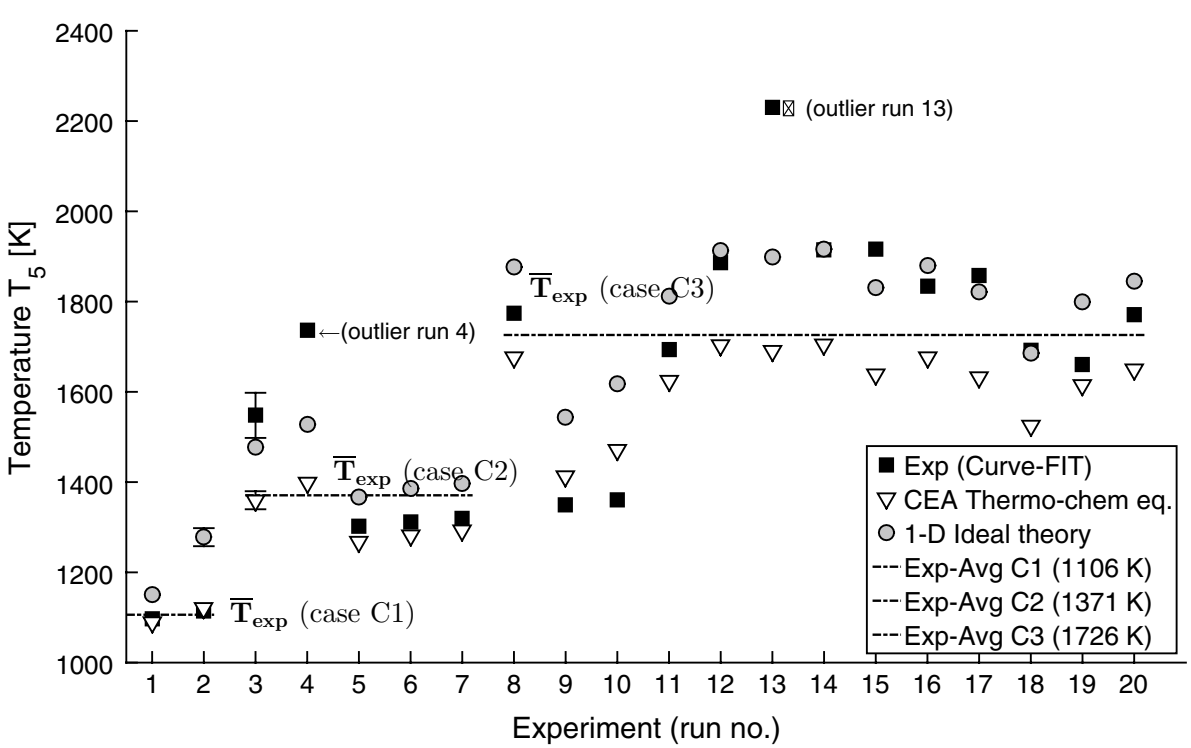


measured by LIGS that are in close agreement to theoretical temperatures $T_{5}$ (th) of 1265-1300 K: reflecting a consistent relative deviation as low as $2.2-2.8 \%$. As runs $5-7$ are thus very consistent, they indicate high accuracy of LIGS when test conditions are well behaved and shot-to-shot variation of diaphragm burst pressure and incident shock Mach number is kept as low as possible to ensure reproducible facility operation. In contrast, variation of diaphragm burst pressure and shock speed can result in evident deviations. However, considering the overall agreement between experiment and theory of below $10 \%$ for the majority of test runs, a relative deviation of beyond $20 \%$, as in the case of test run 4 , is regarded as an evident outlier. This is consistent with an earlier study [20], where intermittent outliers were similarly observed and easily identified as such.

For the third test condition $\mathrm{C} 3$ at the overall highest shock Mach number of 3.5 and the lowest post-reflected shock pressure of $11 \mathrm{MPa}, 13$ measurements (no. 8-20) were conducted. Here, experimental temperatures $T_{5}(\exp )$ measured by single-shot LIGS range from 1350 to $1915 \mathrm{~K}$, where stagnation condition variation is attributed to variation in burst pressure and shock Mach number, the latter ranging from 3.19 to 3.60. Absolute and relative temperature variations between $T_{5}(\mathrm{exp})$ and $T_{5}(\mathrm{th})$ of minimum $46 \mathrm{~K}(2.9 \%)$ for run 19 and maximum $278 \mathrm{~K}(17.0 \%)$ for test run 15 are observed. In contrast, run 13, with a deviation of $540 \mathrm{~K}$ (31.9\%), reflecting an unrealistically high temperature of $2231 \mathrm{~K}$, is easily identified as an outlier and thus omitted from further analysis. Interestingly, the LIGS time oscillation signal for run 13 was by no means of lesser quality, i.e., lower SNR, or of shorter duration than for other test runs (Fig. 8) and was independently confirmed by frequencydomain signal analysis by DFT. Overall, 7 out of 13 LIGS measurements for condition $\mathrm{C} 3$ reflect a relative deviation between $T_{5}(\exp )$ and $T_{5}($ th) of below $10 \%$, whereas five experiments indicated relative deviation between 10 and $20 \%$ from CEA reference predictions.

Finally, the majority of measurements $T_{5}(\exp )$ overpredict theoretical reference temperatures by CEA $T_{5}($ th) by $5-10 \%$, while only a small number of test runs yield measured temperatures lower than CEA. This is in line with earlier observations at lower stagnation temperatures [20], where neither post-processing by time-domain curve fitting nor frequencydomain DFT indicated a systematic bias with respect to reference temperatures.

Yet, it is interesting to note that whereas LIGS almost consistently overpredicts temperatures as predicted by CEA, i.e., assuming thermo-chemical equilibrium, temperatures predicted by Rankine-Hugoniot (shock jump) equations, i.e., 1-D inviscid ideal gas theory, are almost consistently higher than experimental values, see Table 2 . Here, relative deviation of $T_{5}(\exp )$ from $T_{5}($ th) according to ideal gas theory is negative in the majority of cases.
Particularly for case C3, at the overall highest post-shock temperature $1600-1800 \mathrm{~K}$ and lowest stagnation pressure $\sim 11 \mathrm{MPa}$, closer agreement of experiments with theory is obtained when employing ideal gas relations as temperature reference. Specifically, for 8 out of 13 measurements for condition $\mathrm{C} 3$, the relative deviation reduces notably when comparing $T_{5}(\exp )$ against 1-D inviscid theory instead of CEA. For a limited number of four experiments $(12,14,17$, and 18), this reduction is as large as an order of magnitude, where relative deviation reduces from initially above $10 \%$ to now below $5 \%$ (Table 2 ).

Most interestingly, the opposite trend is observed for the undertailored condition $\mathrm{C} 1$, i.e., at the overall lowest post-shock temperature $\sim 1100 \mathrm{~K}$ and highest stagnation pressure of $22 \mathrm{MPa}$ of all test conditions. Here, for both test runs 1 and 2, initially excellent agreement between experiment and theory according to CEA is reflected by a remarkably low relative deviation of well below $1 \%$. This relative deviation rises up to around $-5 \%$ and $-13 \%$ for experiments 1 and 2, respectively, as $T_{5}(\exp )$ is compared with $T_{5}($ th) predicted according to 1-D ideal gas theory, now reflecting an obvious underprediction of reference temperature.

In the case of the intermediate condition $\mathrm{C} 2$, i.e., with post-shock temperature $1300-1500 \mathrm{~K}$ and stagnation pressure $15-16 \mathrm{MPa}$, the identical trend is observed. This observation is, however, less evident which is in part attributed to a larger spread of stagnation quantities for a nominally identical test condition. Both test runs 3 and 4 of lower pressure 13-15 MPa and higher temperature 1600-1700 K reflect relative deviation to CEA temperatures as large as $14 \%$ and $24 \%$ which is reduced to $5 \%$ and $14 \%$, respectively, when compared with ideal gas theory. Hence, even though reference temperature is still consistently overpredicted, absolute and relative deviations reduce notably. By contrast, for three additional test runs 4, 5, and 6 at higher pressure 15-17 $\mathrm{MPa}$ and higher temperature $\sim 1300 \mathrm{~K}$, the CEA temperature was initially closely matched, yet slightly overpredicted, as is reflected by a very consistent relative deviation of $2-3 \%$. As the experimental temperature $T_{5}(\exp )$ is now compared against 1-D ideal theory, relative deviation is observed to consistently rise in magnitude and to turn negative, thus indicating an underprediction of reference temperature by around $-5 \%$.

Average experimental temperatures of $T_{5}(\exp )$ for conditions $\mathrm{C} 1-\mathrm{C} 3$ have been calculated to be $1106 \mathrm{~K}, 1371 \mathrm{~K}$, and $1726 \mathrm{~K}$, respectively. Standard variation from arithmetic average for conditions $\mathrm{C} 1-\mathrm{C} 3$ is calculated to be $1.1 \%, 8.6 \%$, and $11.2 \%$, respectively. Furthermore, the relative standard deviation of incident shock Mach number $M_{\mathrm{s}}$ for conditions $\mathrm{C} 1-\mathrm{C} 3$ is calculated to be $4.5 \%, 3.1 \%$, and $3.7 \%$, respectively, as a measure for test case repeatability. 


\section{Discussion}

LIGS single-shot measurements of the post-reflected shock stagnation temperature presented herein are conducted for a set of three conditions (C1-C3) of constant net burst pressure of $22 \mathrm{MPa}$. By systematic decrease in the shock tube pressure from $0.5 \mathrm{MPa}$ (case $\mathrm{C} 1$ ), $0.25 \mathrm{MPa}$ (case $\mathrm{C} 2$ ) to $0.15 \mathrm{MPa}$ (case C3), incident shock Mach number $M_{\mathrm{s}}$ and post-reflected shock temperature $T_{5}$ are systematically increased, while the stagnation pressure $p_{5}$ is lowered accordingly.

For the majority of measurements of temperature $T_{5}$, good general agreement with theory is found, with relative variation from theoretical values ranging between 1 and $15 \%$. Overall, experimental temperatures $T_{5}(\exp )$ were found to consistently range higher than reference temperatures computed by CEA, assuming full thermo-chemical equilibrium, and to be lower than temperatures calculated according to Rankine-Hugoniot (shock jump) relations of an ideal gas. Against this background, it is interesting to note that, for all 20 single-shot measurements presented herein, a consistent trend in terms of variation of agreement of $T_{5}(\exp )$ with reference temperature $T_{5}(\mathrm{th})$ has been observed, as the post-shock conditions were systematically varied for cases $\mathrm{C} 1-\mathrm{C} 3$. Initially, measurements for condition $\mathrm{C} 1$, at the highest overall pressure and lowest temperature, reflected excellent agreement with CEA, assuming full thermo-chemical equilibrium, whereas agreement with ideal gas theory was much poorer. A systematic decrease in pressure (from initially $22 \mathrm{MPa}$ for $\mathrm{C} 1$ to $11 \mathrm{MPa}$ for $\mathrm{C} 3$ ) and a corresponding increase in stagnation temperature (from initially $1100 \mathrm{~K}$ for $\mathrm{C} 1$ up to $1900 \mathrm{~K}$ for C3) resulted in experimental temperatures $T_{5}(\exp )$ to reflect continually better agreement with ideal gas predictions of $T_{5}($ th $)$ for cases $\mathrm{C} 2$ and C3. Particularly, experimental measurements 8-20 for condition C3 showed much closer agreement with ideal gas theory, as opposed to a notable overprediction of temperatures predicted by CEA.

Considering stagnation temperature below $2000 \mathrm{~K}$ and thus excluding chemical dissociation of $\mathrm{O}_{2}$ or $\mathrm{N}_{2}$, as well as high densities within the nozzle reservoir to induce rapid thermal equilibrium of translational and rotational energies [44], the spread between reference temperatures $T_{5}($ th) calculated according to CEA and ideal gas relations is attributed to an excitation of vibrational degrees of freedom. Hence, the general trend of data to be continuously well represented by reference values by 1-D ideal gas theory and less well captured by CEA predictions, as pressure was decreased and temperature was increased, is indicative of a limited influence of vibrational excitation. For the relevant species and bath gases involved, vibrational relaxation times $\tau_{\text {relax }}$ of collisional partners
$\mathrm{O}_{2}-\mathrm{O}_{2}$ and $\mathrm{N}_{2}-\mathrm{N}_{2}$ and NO- $\mathrm{N}_{2}$ were calculated for stagnation pressures and temperatures of conditions $\mathrm{C} 1-\mathrm{C} 3$ according to the Landau-Teller model [45] and were found in the range of $\tau_{\text {relax }}<10 \mu \mathrm{s}$. In experiments presented in this article, the relative time difference between release of the pump laser pulse and reflected shock passage amounts to $120-150 \mu \mathrm{s}$, depending on incident shock Mach number $M_{\mathrm{s}}$. While this timescale is generally not considered to be of the same order as relevant vibrational relaxation timescales in the high-pressure, high-density post-shock regime, moderate influence due to finite-rate vibrational relaxation is conceivable, yet. This becomes evident when considering the relaxation time $\tau_{\text {relax }}$ to merely represent the time interval, where initial vibrational energy rises by a factor $e$ and does not yet reach its full thermal equilibrium value. The general qualitative trend of vibrational relaxation time $\tau_{\text {relax }}$ to rise with temperature and drop with pressure has been tentatively observed in the experimental data for conditions $\mathrm{C} 1-\mathrm{C} 3$ that is continuously better represented by ideal gas theory than CEA. A similar trend of LIGS single-shot temperature data at lower stagnation pressures to yield better agreement with ideal gas theory than CEA was observed in earlier studies $[20,21]$.

Two out of 20 measurements with individual relative deviation beyond $20 \%$ are clearly identified as outliers. Absolute temperatures derived from the time-domain fit have been independently confirmed by cross-checking with a frequencydomain data analysis by DFT. The most likely cause for such obvious outliers, far from anticipated temperature levels, are transient distortions of the induced density grating. This becomes evident when considering temperatures to be extracted from single-shot measurements, i.e., by induction of a local density grating into a highly perturbed flow, as present in the stagnated endwall region. However, in addition to grating perturbations by laser beam steering and deflection in the wake of the reflected shock front, as reported by Mizukaki and Matsuzawa [27], some of these fluctuations pertain to variation of the stagnated gas properties, as induced by secondary compression and expansion waves within the shock tube. Such undesirable waves are typically caused by a free-piston driver which is not exactly tuned, as in the present study, and propagate downstream to the nozzle reservoir. Further influence can result from under- and overtailoring where expansion and compression waves between the contact surface and ST-endwall can entail distortions of the transient LIGS grating. Therefore, operating short-duration facility with a tuned piston driver and in tailored mode is considered to be generally beneficial in order to avoid LIGS single-shot measurements to be affected by transient fluctuations. However, as has been demonstrated by the majority of successful measurements presented herein, LIGS single-shot measurements of stagnation temperature within the nozzle reservoir are also possible without tuned piston and tailored interface operation. Accordingly, 
the relatively large scatter observed, particularly for conditions $\mathrm{C} 2$ and $\mathrm{C} 3$, is not primarily due to random variation of LIGS single-shot measurements, but attributed to variation of main diaphragm burst pressure and hence incident shock Mach number $M_{\mathrm{s}}$. This is exemplified by an inherent relative standard deviation of $M_{\mathrm{s}}$ between 3.1 and $4.5 \%$ for cases $\mathrm{C} 1-\mathrm{C} 3$ and associated experimental uncertainties. Besides influence from inherent shot-to-shot variation, experimental scatter observed can further be affected by non-ideal temporal variation of postreflected shock conditions, estimated less than $2 \%$ for temperature $T_{5}$ at test time instant according to Peterson and Hanson [8]. Similar findings were reported by Richter et al. [46] who observed a negative frequency chirp, i.e., temporally decreasing signal frequency, that is indicative of gradually decreasing sound speed and temperature in the post-reflected shock regime, even for tailored interface conditions.

Hence, single-shot uncertainty of measurements presented herein is inferred to be dominated by reasonable repeatability, i.e., precision, due to facility-inherent shotto-shot variation rather than being limited by systematic offset, i.e., lack in accuracy. As such, when excluding those evident outliers from statistical analysis of an otherwise reproducible test condition, single-shot measurements have been demonstrated to be well correlated with the mean value [20]. Under idealized conditions in a quiescent test chamber, LIGS single-shot precision of the current set-up was determined to be as low as $3 \%$ by Sander et al. [23].

In comparison, prior broadband coherent anti-Stokes Raman scattering (CARS) measurements of rotational and vibrational temperatures in hypersonic freestream and behind bow shock layers by Boyce et al. [1] and Pulford et al. [8] reported single-shot uncertainties, i.e., relative deviation, as large as $10 \%$, thus of same order of magnitude as LIGS single-shot measurements presented herein. However, as stated in the Introduction, application of CARS measurements to in situ nozzle reservoir thermometry is deemed impractical due to high density and pressure in the postshock regime. Hence, the positive correlation of LIGS signal intensity with pressure is considered particularly useful, as it complements the range of established optical diagnostics by a technique, viable for remote temperature measurements under high-pressure conditions. It is thus particularly useful for application to short-duration ground test facilities. This is demonstrated by experiments presented herein to represent the highest pressures and temperatures measured by LIGS in the literature, to the best knowledge of the authors.

\section{Conclusion}

In order to contribute to an increasingly accurate determination of nozzle inlet conditions of short-duration test facilities, non-intrusive measurements of post-reflected shock stagnation temperature in situ within the nozzle reservoir of the free-piston shock tunnel HELM have been performed by resonant homodyne LIGS. Twenty single-shot measurements are presented for three representative, under-/overtailored test conditions in air, at low/medium enthalpy (1.2-2.1 MJ/ $\mathrm{kg}$ ) in the temperature and pressure range of 1100-1900 K and 9-22 MPa. Experimental data are compared with theoretical reference to within $1-15 \%$, reflecting reasonable scatter due to shot-to-shot variation. With increasing temperature and decreasing pressure, measurements are observed to be increasingly better represented by ideal gas 1-D shock jump relations rather than full thermo-chemical equilibrium predictions. This trend may be indicative of a limited influence of finite-rate vibrational relaxation.

Advancement of the optical set-up by adjustment of the laser beam path, revision of the optical access to the nozzle reservoir, and limitation of facility recoil by a hydraulic damping system were shown to significantly enhance robustness of the measurement technique, indicated by a significantly improved success rate of near $90 \%$. LIGS was demonstrated to enable single-shot temperature determination at elevated pressures within a full-scale, short-duration, hypersonic facility at increasingly ground testing-relevant operation conditions. Due to positive correlation of signal intensity with pressure and resolved prior mechanical limitations of the HELM facility, increasing gas temperatures are not considered to be a limiting factor for application of LIGS to higher temperature and enthalpy test conditions in future.

Funding Open Access funding enabled and organized by Projekt DEAL.

Open Access This article is licensed under a Creative Commons Attribution 4.0 International License, which permits use, sharing, adaptation, distribution and reproduction in any medium or format, as long as you give appropriate credit to the original author(s) and the source, provide a link to the Creative Commons licence, and indicate if changes were made. The images or other third party material in this article are included in the article's Creative Commons licence, unless indicated otherwise in a credit line to the material. If material is not included in the article's Creative Commons licence and your intended use is not permitted by statutory regulation or exceeds the permitted use, you will need to obtain permission directly from the copyright holder. To view a copy of this licence, visit http://creativecommons.org/licenses/by/4.0/.

\section{References}

1. Danehy, P.M., Bathel, B.F., Johansen, C.T., Winter, M., O'Byrne, S., Cutler, A.D.: Molecular-based optical diagnostics for hypersonic nonequilibrium flows. In: Josyula, E. (ed.) Hypersonic Nonequilibrium Flows: Fundamentals and Recent Advances, pp. 343370. AIAA Inc., Reston (2015). https://doi.org/10.2514/5.97816 24103292.0343.0470 
2. Olivier, H.: An improved method to determine free stream conditions in hypersonic facilities. Shock Waves 3, 129-139 (1993). https://doi.org/10.1007/BF02115892

3. McGilvray, M., Dann, A.G., Jacobs, P.A.: Modelling the complete operation of a free-piston shock tunnel for a low enthalpy condition. Shock Waves 23, 399-406 (2013). https://doi.org/10.1007/ s00193-013-0437-8

4. Esser, B.: Die Zustandsgrößen im Stoßwellenkanal als Ergebnis eines exakten Riemannlösers. PhD Thesis, Shock Wave Laboratory, RWTH-Aachen University, Aachen (1991)

5. MacLean, M., Candler, G., Holden, M.S.: Numerical evaluation of flow conditions in the LENS reflected shock-tunnel facilities. 43rd AIAA Aerospace Sciences Meeting and Exhibit, Reno, NV, AIAA Paper 2005-903 (2005). https://doi.org/10.2514/6.2005-903

6. Stalker, R.J., Crane, K.C.A.: Driver gas contamination in a highenthalpy reflected shock tunnel. AIAA J. 16, 277-279 (1978). https://doi.org/10.2514/3.7520

7. Monson, D.J.: Measurements of the duration of constant reflectedshock temperature in a reflected-shock tunnel. AIAA J. 9, 18721874 (1971). https://doi.org/10.2514/3.6440

8. Peterson, E.L., Hanson, R.K.: Nonideal effects behind reflected shock waves in a high-pressure shock tube. Shock Waves 10, 405-420 (2001). https://doi.org/10.1007/PL00004051

9. Hornung, H.G., Belanger, J.: Role techniques of ground testing for simulation of flows up to orbital speeds. 16th Aerodynamic Ground Testing Conference, Seattle, WA, AIAA Paper 19901377 (1990). https://doi.org/10.2514/6.1990-1377

10. Boyce, R., Pulford, D., Houwing, A., Mundt, Ch.: Rotational and vibrational temperature measurements using CARS in a hypervelocity shock layer flow and comparisons with CFD calculations. Shock Waves 6, 41-51 (1996). https://doi. org/10.1007/BF02511403

11. Eckbreth, A.C.: Laser Diagnostics for Combustion Temperature and Species. Gordon and Breach, New York (1996)

12. Pulford, D., Newman, D.S., Houwing, A., Sandeman, R.J.: The application of coherent anti-stokes raman scattering to temperature measurements in a pulsed high enthalpy supersonic flow. Shock Waves 4, 119-125 (1994). https://doi.org/10.1007/BF014 17427

13. Grisch, F., Bouchardy, P., Clauss, W.: CARS thermometry in high pressure rocket combustors. Aerosp. Sci. Technol. 7, 317-330 (2003). https://doi.org/10.1016/S1270-9638(03)00017-8

14. Danehy, P.M., Palma, P., Houwing, A., McIntyre, T.: Comparison of theoretical laser-induced fluorescence images with measurements performed in a hypersonic shock tunnel. AIAA Advanced Measurement and Ground Testing Technology Conference, New Orleans, LA, AIAA Paper 96-2236 (1996). https://doi. org/10.2514/6.1996-2236

15. Sutton, K.S., Graves, R.A.: A general stagnation-point convective-heating equation for artibrary gas mixtures. NASA-TR-R-376 (1971). https://ntrs.nasa.gov/api/citations/19720003329/downl oads/19720003329.pdf

16. Palmer, J.L., McMillin, B.K., Hanson, R.K.: Planar laserinduced fluorescence imaging of velocity and temperature in shock tunnel free jet flow. 30th Aerospace Sciences Meeting \& Exhibit, Reno, NV, AIAA Paper 1992-762 (1992). https://doi. org/10.2514/6.1992-762

17. Laux, C.O., Spence, T.G., Kruger, C.H., Zare, R.N.: Optical diagnostics of atmospheric air plasmas. Plasma Sources Sci. Technol. 12, 125-138 (2003). https://doi.org/10.1088/0963-0252/12/2/301

18. Parker, R.A., Wakeman, T., MacLean, M., Holden, M.S.: Measuring nitric oxide freestream concentration using quantum cascade lasers at CUBRC. 44th AIAA Aerospace Sciences Meeting and Exhibit, Reno, NV, AIAA Paper 2006-926 (2006). https://doi. org/10.2514/6.2006-926
19. Sander, T., Altenhoefer, P., Mundt, Ch.: Temperature measurements in a shock tube using laser-induced grating spectroscopy. AIAA J. Thermophys. Heat Transf. 30, 62-66 (2016). https://doi. org/10.2514/1.T4556

20. Selcan, C., Sander, T., Altenhoefer, P., Koroll, F., Mundt, Ch.: Stagnation temperature measurements in a shock-tunnel facility using laser-induced grating spectroscopy. AIAA J. Thermophys. Heat Transf. 32, 226-236 (2017). https://doi.org/10.2514/1.T5199

21. Altenhoefer, P., Sander, T., Koroll, F., Mundt, Ch.: LIGS measurements in the nozzle reservoir of a free piston shock tunnel. Shock Waves 29, 307-320 (2017). https://doi.org/10.1007/s0019 3-018-0808-2

22. Danehy, P.M., Paul, H.P., Farrow, R.L.: Thermal-grating contributions to degenerate four-wave mixing in nitric oxide. J. Opt. Soc. Am. B 12, 1564-1576 (1995). https://doi.org/10.1364/JOSAB .12 .001564

23. Sander, T., Altenhoefer, P., Mundt, Ch.: Development of laserinduced grating spectroscopy for application in shock tunnels. AIAA J. Thermophys. Heat Transf. 28, 27-31 (2014). https://doi. org/10.2514/1.T4131

24. Latzel, H., Dreizler, A., Dreier, T., Heinze, J., Dillmann, M., Stricker, W., Lloyd, G.M., Ewart, P.: Thermal grating and broadband degenerate four-wave mixing spectroscopy of $\mathrm{OH}$ in highpressure flames. Appl. Phys. B 67, 667-673 (1998). https://doi. org/10.1007/s003400050563

25. McIntyre, T., Kleine, H., Houwing, A.F.P.: Optical imaging techniques for hypersonic impulse facilities. Aeronaut. J. 111(1115), 1-16 (2007). https://doi.org/10.1017/S0001924000001718

26. Cummings, E.B., Leyva, I.A., Hornung, H.G.: Laser-induced thermal acoustics (LITA) signals from finite beams. Appl. Opt. 34, 3290-3302 (1995). https://doi.org/10.1364/AO.34.003290

27. Mizukaki, T., Matsuzawa, T.: Application of laser-induced thermal acoustics in air to measurement of shock-induced temperature changes. Shock Waves 19, 361-369 (2009). https://doi. org/10.1007/s00193-009-0218-6

28. Förster, F.J., Baab, S., Lamanna, G., Weigand, B.: Temperature and velocity determination of shock-heated flows with non-resonant heterodyne laser-induced thermal acoustics. Appl. Phys. B 121, 235-248 (2015). https://doi.org/10.1007/s00340-015-6217-7

29. Cummings, E.B.: Laser-induced thermal acoustics: simple accurate gas measurements. Opt. Lett. 19, 1361-1363 (1994). https:// doi.org/10.1364/OL.19.001361

30. Eichler, H.J., Günter, P., Pohl, D.W.: Laser-Induced Dynamic Gratings. Springer, New York (1986)

31. Schlamp, S., Cummings, E.B., Hornung, H.G.: Beam misalignments and fluid velocities in laser-induced thermal acoustics. Appl. Opt. 38, 5724-5733 (1999). https://doi.org/10.1364/ AO.38.005724

32. Mundt, Ch., Altenhoefer, P.: Initial results of HELM in initial operation as shock-tube. 11th International Workshop on Shock Tube Technology, Australian Hypersonics Workshop 2011, Brisbane (2011)

33. Schemperg, K.A., Mundt, Ch.: Study of numerical simulations for optimized operation of the free piston shock tunnel HELM. 15th AIAA International Space Planes and Hypersonic Systems and Technologies Conference, Dayton, OH, AIAA Paper 2008-2653 (2008). https://doi.org/10.2514/6.2008-2653

34. Schemperg, K.A.: Gasdynamische Auslegung und Entwicklung des kolbengetriebenen Stoßwellenkanals HELM. Ph.D. Thesis, Department of Aerospace Engineering, University of the Federal Armed Forces Munich, Munich (2009) (in German)

35. Schneider, W., Moortgat, G.K., Tyndall, G.S., Burrows, J.P.: Absorption cross-sections of $\mathrm{NO}_{2}$ in the $\mathrm{UV}$ and visible region (200-700 nm) at 298 K. J. Photochem. Photobiol. A 40, 195-217 (1987). https://doi.org/10.1016/1010-6030(87)85001-3 
36. Hsu, D.K., Monts, D.L., Zare, R.N.: Spectral Atlas of Nitrogen Dioxide 5330 to $6480 \AA$ A. Academic Press, New York (1978)

37. Schlamp, S., Rösgen, T., Kozlov, D.N., Rakut, C., Kasal, P., von Wolfersdorf, J.: Transient grating spectroscopy in a hot turbulent compressible free jet. J. Propul. Power 21, 1008-1018 (2005). https://doi.org/10.2514/1.13794

38. Lemmon, E.W., Jacobsen, R.T., Penoncello, S.G., Friend, D.G.: Thermodynamic properties of air and mixtures of nitrogen, argon, and oxygen from 60 to $2000 \mathrm{~K}$ at pressures to $2000 \mathrm{MPa}$. J. Chem. Phys. Reference Data 29, 331-386 (2000). https://doi. org/10.1063/1.1285884

39. Gordon, S., McBride, B.J.: Computer program for calculation of complex chemical equilibrium compositions and applications, I: Analysis, II: Users manual and program description. NASA Reference Publication 1311 (1994). https://ntrs.nasa.gov/api/citat ions/19950013764/downloads/19950013764.pdf

40. Jacobs, P.A.: Quasi-one-dimensional modeling of a freepiston shock tunnel. AIAA J. 32, 137-145 (1994). https://doi. org/10.2514/3.11961

41. Mundt, Ch., Boyce, R., Jacobs, P.A., Hannemann, K.: Validation study of numerical simulations by comparison to measurements in piston-driven shock-tunnels. Aerosp. Sci. Technol. 11, 100-109 (2007). https://doi.org/10.1016/j.ast.2006.12.002
42. Schlamp, S., Hornung, H.G., Sobota, T.H., Cummings, E.B.: Accuracy and uncertainty of single-shot, nonresonant laserinduced thermal acoustics. Appl. Opt. 39, 5477-5481 (2000). https://doi.org/10.1364/AO.39.005477

43. Kline, S.J., McClintock, F.A.: Describing uncertainties in single sample experiments. Mech. Eng. 75, 3-8 (1953)

44. Blackman, V.: Vibrational relaxation in oxygen and nitrogen. J. Fluid Mech. 1, 61-85 (1956). https://doi.org/10.1017/S002211205 6000056

45. Vincenti, W.G., Kruger, C.H.: Introduction to Physical Gas Dynamics. Krieger Publishing Company, New York (1977)

46. Richter, J., Mayer, J., Weigand, B.: Accuracy of non-resonant laser-induced thermal acoustics (LITA) in a convergent-divergent nozzle flow. Appl. Phys. B 124(19), 1-12 (2018). https:// doi.org/10.1007/s00340-017-6885-6

Publisher's Note Springer Nature remains neutral with regard to jurisdictional claims in published maps and institutional affiliations. 